\title{
Bianchi identities in higher dimensions
}

\author{
V. Pravda †, A. Pravdováł, A. Coley $\ddagger$, R. Milson $\ddagger$ \\ † Mathematical Institute, Academy of Sciences, Žitná 25, 11567 Prague 1, \\ Czech Republic \\ $\ddagger$ Dept. Mathematics and Statistics, Dalhousie U., Halifax, Nova Scotia B3H \\ 3J5, Canada \\ E-mail: pravda@math.cas.cz, pravdova@math.cas.cz, aac@mathstat.dal.ca, \\ milson@mathstat.dal.ca
}

\begin{abstract}
A higher dimensional frame formalism is developed in order to study implications of the Bianchi identities for the Weyl tensor in vacuum spacetimes of the algebraic types III and $\mathrm{N}$ in arbitrary dimension $n$. It follows that the principal null congruence is geodesic and expands isotropically in two dimensions and does not expand in $n-4$ spacelike dimensions or does not expand at all. It is shown that the existence of such principal geodesic null congruence in vacuum (together with an additional condition on twist) implies an algebraically special spacetime. We also use the Myers-Perry metric as an explicit example of a vacuum type $\mathrm{D}$ spacetime to show that principal geodesic null congruences in vacuum type D spacetimes do not share this property.
\end{abstract}

\section{Introduction}

For dimensions $n<4$ the Weyl tensor vanishes identically and for $n=4$ it has very special properties. It is of interest to determine which of the properties of fourdimensional (4D) spacetimes can be straightforwardly generalized to higher dimensions and which need to be modified or do not hold at all.

Recently a classification of algebraic tensor types in Lorentzian manifolds of arbitrary dimension was developed [1. For the Weyl tensor in 4D this classification reproduces the Petrov classification and for the Ricci tensor in 4D the Segre classification.

In $4 \mathrm{D}$ it follows from the Bianchi identitiest

$$
R_{a b\{c d ; e\}}=0
$$

that in algebraically special vacuum spacetimes the multiple principal null direction of the Weyl tensor is geodesic and shearfree. In this paper a higher dimensional frame formalism is developed in order to study implications of the Bianchi identities, which are given in Appendix B for vacuum spacetimes of algebraic types N and III in higher dimensions. Although in most applications it is necessary to perform calculations in a given spacetime dimension $n$, in this paper we present results without specifying the dimension and hence these results are valid in any dimension.

$\ddagger$ Note that in this paper we use two different operations denoted by \{\} . In the first case \{\} acts on three indices and stands for $R_{a b\{c d ; e\}}=R_{a b c d ; e}+R_{a b d e ; c}+R_{a b e c ; d}$. In the other case \{\} acts on four indices and is given by (5). 
In $4 \mathrm{D}$, for algebraically special vacuum spacetimes some of the tetrad components of the Bianchi identities in the Newman-Penrose formalism [2] lead to simple algebraic equations (i.e. equations with no derivatives). In higher dimensions these algebraic equations are much more complex and the number of independent equations, as well as the number of unknowns, depend on the dimension of the spacetime.

We show that in vacuum type III and $\mathrm{N}$ spacetimes of arbitrary dimension the multiple principal null direction (PND) is geodesic. For type N spacetimes (Sec. 3) the symmetric expansion matrix $\boldsymbol{S}$ has just one non-vanishing doubly degenerate eigenvalue. Consequently, the principal geodesic null congruence expands isotropically in two dimensions and does not expand in $n-2$ dimensions. Thus, shear does not vanish for $n>4$. The antisymmetric twist matrix $\boldsymbol{A}$ has only one independent component. All other components may be set to zero by appropriately choosing the frame. For type III, we prove similar results in the generic case (Sec. 4) and in the non-twisting case Appendix C.1. The complete proof, including all possible degenerate cases, is presented for five-dimensional spacetimes Appendix C.2.

In Section [5] we show that for a vacuum spacetime the properties of $\boldsymbol{S}$ and $\boldsymbol{A}$ matrices mentioned above imply that the spacetime is algebraically special. We also show that an arbitrary vacuum spacetime admitting a non-expanding and non-twisting geodesic null congruence (i.e., a higher dimensional generalization of the vacuum Kundt class) is algebraically special.

These statements cannot be regarded as a generalization of the Goldberg-Sachs theorem for higher dimensions (see Sec. 5 for details) since in Appendix D it is shown that the Myers-Perry metric, which is of the algebraic type D, has the expansion matrix $\boldsymbol{S}$ with one doubly degenerate eigenvalue and also another non-vanishing eigenvalue.

In Section 6, we conclude with discussion of potential applications.

\section{Preliminaries}

The Newman-Penrose formalism 2] in 4D is based on using a null tetrad $\boldsymbol{\ell}, \boldsymbol{n}, \boldsymbol{m}$ and $\overline{\boldsymbol{m}}$, where $\boldsymbol{\ell}$ and $\boldsymbol{n}$ are real null vectors and $\boldsymbol{m}$ and $\overline{\boldsymbol{m}}$ are complex null vectors instead of an orthonormal basis, thus taking advantage of the null cone structure of spacetimes. For $n$-dimensional calculations it seems to be more practical to choose a pair of null vectors $\boldsymbol{\ell}, \boldsymbol{n}$ and an orthonormal set of real spacelike vectors $\boldsymbol{m}^{(i)}$. We thus need two types of indices: indices $a, b, \ldots$ with values $0, \ldots, n-1$ and indices $i, j, \ldots$ going from 2 to $n-1$. We will observe Einstein's summation convention for both of these types of indices. However, for indices $i, j, \ldots$, there is no difference between covariant and contravariant components an thus we will not distinguish between subscripts and superscripts.

The frame

$$
\boldsymbol{m}^{(0)}=\boldsymbol{n}, \boldsymbol{m}^{(1)}=\boldsymbol{\ell}, \boldsymbol{m}^{(i)}
$$

thus satisfies

$$
\ell^{a} \ell_{a}=n^{a} n_{a}=\ell^{a} m_{a}^{(i)}=n^{a} m_{a}^{(i)}=0, \quad \ell^{a} n_{a}=1, \quad m^{(i) a} m_{a}^{(j)}=\delta_{i j},
$$

and the metric has the form

$$
g_{a b}=2 \ell_{(a} n_{b)}+\delta_{i j} m_{a}^{(i)} m_{b}^{(j)} .
$$

If one would like to completely generalize the Newman-Penrose formalism for higher dimensions it would be necessary to denote Ricci rotation-coefficients and all 
independent components of the Riemann, Weyl and Ricci tensors and then rewrite all frame components of the Bianchi identities (11) and the Ricci identities

$$
V_{a ; b c}=V_{a ; c b}+R_{a b c}^{s} V_{s},
$$

where $\boldsymbol{V}$ is an arbitrary vector, as well as expressions for commutators 8 . of covariant derivatives in directions of the frame vectors

$$
D \equiv \ell^{a} \nabla_{a}, \quad \triangle \equiv n^{a} \nabla_{a}, \quad \delta_{i} \equiv m^{(i) a} \nabla_{a} .
$$

However, in this paper we are only interested in studying consequences of the Bianchi identities, which are given in detail in Appendix B, and thus we do not introduce the Ricci identities except for equation (103) given in Section 5 ,

\subsection{Decomposition of the Riemann and Weyl tensors}

In order to construct a basis in the space of 4-rank tensors with symmetries

$$
R_{a b c d}=\frac{1}{2}\left(R_{[a b][c d]}+R_{[c d][a b]}\right),
$$

we introduce the operation \{\}

$$
w_{\{a} x_{b} y_{c} z_{d\}} \equiv \frac{1}{2}\left(w_{[a} x_{b]} y_{[c} z_{d]}+w_{[c} x_{d]} y_{[a} z_{b]}\right) .
$$

When decomposing the Riemann tensor in terms of the frame vectors we also have to take into account that

$$
R_{a\{b c d\}}=0 .
$$

Now let us decompose the Riemann tensor in its frame components and sort them by their boost weights (see [1]):

$$
\begin{aligned}
& R_{a b c d}=\overbrace{4 R_{0 i 0 j} n_{\{a} m_{b}^{(i)} n_{c} m_{d\}}^{(j)}}^{2} \\
& +\overbrace{8 R_{010 i} n_{\{a} \ell_{b} n_{c} m_{d\}}^{(i)}+4 R_{0 i j k} n_{\{a} m_{b}^{(i)} m_{c}^{(j)} m_{d\}}^{(k)}}^{1} \\
& \left.+4 R_{0101} n_{\{a} \ell_{b} n_{c} \ell_{d\}}+4 R_{01 i j} n_{\{a} \ell_{b} m_{c}^{(i)} m_{d\}}^{(j)} \quad\right\}^{0} \\
& \left.+8 R_{0 i 1 j} n_{\{a} m_{b}^{(i)} \ell_{c} m_{d\}}^{(j)}+R_{i j k l} m_{\{a}^{(i)} m_{b}^{(j)} m_{c}^{(k)} m_{d\}}^{(l)}\right\} \\
& +\overbrace{8 R_{101 i} \ell_{\{a} n_{b} \ell_{c} m_{d\}}^{(i)}+4 R_{1 i j k} \ell_{\{a} m_{b}^{(i)} m_{c}^{(j)} m_{d\}}^{(k)}}^{-1} \\
& +\overbrace{4 R_{1 i 1 j} \ell_{\{a} m_{b}^{(i)} \ell_{c} m_{d\}}^{(j)}}^{-2} .
\end{aligned}
$$

The Riemann frame components in this relation are subject to constraints following from (4) and (6),

$$
\begin{aligned}
& R_{0[i|0| j]}=0 \\
& R_{0 i(j k)}=R_{0\{i j k\}}=0, \\
& R_{i j k l}=R_{\{i j k l\}}, \quad R_{i\{j k l\}}=0, \quad R_{01 i j}=2 R_{0[i|1| j]}, \\
& R_{1 i(j k)}=R_{1\{i j k\}}=0, \\
& R_{1[i|1| j]}=0 .
\end{aligned}
$$


Let us check that we have an appropriate number of independent frame components. It is well known that an $n$-dimensional Riemann tensor has

$$
\frac{n^{2}\left(n^{2}-1\right)}{12}
$$

independent components. Counting independent frame components of various boost weights, we obtain

$2 \overbrace{\left(\frac{m(m+1)}{2}\right)}^{2,-2}+2 \overbrace{\left(\frac{(m+1) m(m-1)}{3}+m\right)}^{1,-1}+\overbrace{\frac{m^{2}\left(m^{2}-1\right)}{12}+m^{2}+1}^{0}$,

where $m=n-2$. This is in agreement with (8).

Similarly, it is possible to decompose the Weyl tensor but due to its tracelessness we have the following additional conditions:

$$
\begin{aligned}
& C_{0 i 0 i}=C_{1 i 1 i}=0, \\
& C_{010 i}=C_{0 j i j}, \quad C_{101 i}=C_{1 j i j}, \\
& 2 C_{0 i 1 j}=C_{01 i j}-C_{i k j k}, \quad C_{0101}=-\frac{1}{2} C_{i j i j} .
\end{aligned}
$$

It is well known that an $n$-dimensional Weyl tensor has

$$
\frac{(n+2)(n+1) n(n-3)}{12}
$$

independent components. By counting the independent scalars of various boost weights, we obtain

$\overbrace{2\left(\frac{(m+2)(m-1)}{2}\right)}^{2,-2}+\overbrace{2\left(\frac{(m+1) m(m-1)}{3}\right)}^{1,-1}+\overbrace{\frac{m^{2}\left(m^{2}-1\right)}{12}+\frac{m(m-1)}{2}}^{0}$,

which is in agreement with (10).

The primary algebraic classification of the Weyl tensor in higher dimensions [1] is based on whether all Weyl frame components of a boost weight higher then a specified number can be transformed away by an appropriate choice of the null direction $\boldsymbol{\ell}$. If it is indeed possible, we call the corresponding direction a Weyl aligned null direction (WAND) of an appropriate order $(0,1,2,3)$. If the set of WANDs of a given order is discrete, we call the corresponding directions principal null directions (PND).

Type III and N spacetimes admitting a WAND of order 2 and 3, respectively, were introduced in [7. We will use the notation based on that given in [7] which is suitable for these algebraic classes but becomes rather cumbersome in more general cases. Let us in accordance with [1, 7] state that a spacetime is of the algebraic type III if there exists a frame (2) in which the Weyl tensor has the form

$C_{a b c d}=8 \Psi_{i} \ell_{\{a} n_{b} \ell_{c} m_{d\}}^{(i)}+8 \Psi_{i j k} m_{\{a}^{(i)} m_{b}^{(j)} \ell_{c} m_{d\}}^{(k)}+8 \Psi_{i j} \ell_{\{a} m_{b}^{(i)} \ell_{c} m_{d\}}^{(j)}$,

with $\Psi_{i j k} \neq 0$. The case with $\Psi_{i j k}=0$ (and consequently from (12) also $\Psi_{i}=0$ ) is of the algebraic type $\mathrm{N}$. Note, however, that in this paper the operation \{\} differs by a factor $1 / 8$ from that given in 7 .

The components of the Weyl tensor $\Psi_{i}, \Psi_{i j k}$ and $\Psi_{i j}$ are given by

$$
\Psi_{i}=C_{101 i}, \quad \Psi_{i j k}=\frac{1}{2} C_{1 k i j}, \quad \Psi_{i j}=\frac{1}{2} C_{1 i 1 j} .
$$


Note that $\Psi_{i j}$ is symmetric and traceless. $\Psi_{i j k}$ is antisymmetric in the first two indices and from (7) and (9) it also follows that

$$
\begin{aligned}
& \Psi_{i}=2 \Psi_{i j j}, \\
& \Psi_{\{i j k\}}=0 .
\end{aligned}
$$

\subsection{Decomposition of covariant derivatives of the frame vectors}

Let us denote components of covariant derivatives of the frame vectors $\boldsymbol{\ell}, \boldsymbol{n}, \boldsymbol{m}^{(i)}$ by $L_{a b}, N_{a b}$ and $\stackrel{i}{M}$ ab , respectively,

$\ell_{a ; b}=L_{c d} m_{a}^{(c)} m_{b}^{(d)}, \quad n_{a ; b}=N_{c d} m_{a}^{(c)} m_{b}^{(d)}, \quad m_{a ; b}^{(i)}=\stackrel{i}{M_{c d}} m_{a}^{(c)} m_{b}^{(d)}$.

Since the norm of all frame vectors is constant, it follows that

$$
L_{0 a}=N_{1 a}=\stackrel{i}{M}_{i a}=0 .
$$

Also from the fact that all scalar products of the frame vectors are constant, we get

$$
N_{0 a}+L_{1 a}=0, \quad \stackrel{i}{M}_{0 a}+L_{i a}=0, \quad \stackrel{i}{M_{1 a}}+N_{i a}=0, \quad \stackrel{i}{M}_{j a}+\stackrel{j}{M}_{i a}=0 .
$$

We thus arrive at

$$
\begin{aligned}
\ell_{a ; b}= & L_{11} \ell_{a} \ell_{b}+L_{10} \ell_{a} n_{b}+L_{1 i} \ell_{a} m_{b}^{(i)}+L_{i 1} m_{a}^{(i)} \ell_{b}+L_{i 0} m_{a}^{(i)} n_{b}+L_{i j} m_{a}^{(i)} m_{b}^{(j)} \\
n_{a ; b}= & -L_{11} n_{a} \ell_{b}-L_{10} n_{a} n_{b}-L_{1 i} n_{a} m_{b}^{(i)}+N_{i 1} m_{a}^{(i)} \ell_{b}+N_{i 0} m_{a}^{(i)} n_{b}+N_{i j} m_{a}^{(i)} m_{b}^{(j)} \\
m_{a ; b}^{(i)}= & -N_{i 1} \ell_{a} \ell_{b}-N_{i 0} \ell_{a} n_{b}-L_{i 1} n_{a} \ell_{b}-L_{i 0} n_{a} n_{b}-N_{i j} \ell_{a} m_{b}^{(j)} \\
& \quad \stackrel{i}{M_{j 1}} m_{a}^{(j)} \ell_{b}-L_{i j} n_{a} m_{b}^{(j)}+\stackrel{i}{M_{j 0}} m_{a}^{(j)} n_{b}+M_{k l} m_{a}^{(k)} m_{b}^{(l)}
\end{aligned}
$$

\subsection{Null geodesic congruences}

In Sections 3 and 4, we will show that the multiple PND in type III and N vacuum spacetimes is geodesic. Let us thus study properties of null geodesic congruences in higher dimensions. Analogous but in some cases not fully equivalent definitions are given in the Appendix of the paper [10].

The congruence corresponding to $\boldsymbol{\ell}$ is geodesic if

$$
\ell_{a ; b} \ell^{b} \propto \ell_{a} \Leftrightarrow L_{i 0}=0
$$

It is always possible to rescale $\ell$ (and consequently also $\boldsymbol{n}$ ) in such a way that $\ell_{a ; b} \ell^{b}=0$ and thus also $L_{10}=0$. From now on we will use this parametrization. Then the covariant derivative of the vector $\ell$ is

$$
\ell_{a ; b}=L_{11} \ell_{a} \ell_{b}+L_{1 i} \ell_{a} m_{b}^{(i)}+L_{i 1} m_{a}^{(i)} \ell_{b}+L_{i j} m_{a}^{(i)} m_{b}^{(j)} .
$$

Let us decompose $\boldsymbol{L}$ into its symmetric and antisymmetric parts, $\boldsymbol{S}$ and $\boldsymbol{A}$,

$$
L_{i j}=S_{i j}+A_{i j}, \quad S_{i j}=S_{j i}, A_{i j}=-A_{j i},
$$

where

$$
S_{i j}=\ell_{(a ; b)} m^{(i) a} m^{(j) b}, \quad A_{i j}=\ell_{[a ; b]} m^{(i) a} m^{(j) b} .
$$

We define the expansion $\theta$ and the shear matrix $\sigma_{i j}$ as follows:

$$
\begin{aligned}
\theta & \equiv \frac{1}{n-2} \ell_{; a}^{a}=\frac{1}{n-2}[\boldsymbol{S}], \\
\sigma_{i j} & \equiv\left(\ell_{(a ; b)}-\theta \delta_{k l} m_{a}^{(k)} m_{b}^{(l)}\right) m^{(i) a} m^{(j) b}=S_{i j}-\frac{[\boldsymbol{S}]}{n-2} \delta_{i j} .
\end{aligned}
$$


We will also denote $\sigma^{2} \equiv \sigma_{i j} \sigma_{i j}$. Let us, for simplicity, call $\boldsymbol{A}$ the twist matrix and $\boldsymbol{S}$ the expansion matrix, though $\boldsymbol{S}$ contains information about both, expansion and shear. For simplicity we also introduce quantities

$$
S \equiv \frac{1}{2}[\boldsymbol{S}], \quad A^{2} \equiv \frac{1}{2} A_{i j} A_{i j}
$$

\section{Type $\mathrm{N}$ vacuum spacetimes}

For type N spacetimes the Weyl tensor (11) has the form

$$
C_{a b c d}=8 \Psi_{i j} \ell_{\{a} m_{b}^{(i)} \ell_{c} m_{d\}}^{(j)},
$$

where $\boldsymbol{\Psi}$ is symmetric and traceless. Bianchi equations, given in Appendix B, now reduce to

$$
\begin{aligned}
D \Psi_{i j} & =-2 \Psi_{k(i} \stackrel{k}{M} \stackrel{s}{j) 0}-\Psi_{i k} L_{k j}-2 \Psi_{i j} L_{10}, \\
\delta_{[k} \Psi_{j] i} & =-\Psi_{i s} \stackrel{s}{M}[j k] \\
0 & =L_{k[i} \Psi_{j] k}, \\
0 & =\Psi_{i[j} L_{k] 0}, \\
0 & =L_{k[j} \Psi_{m] i}+\Psi_{i[j} L_{k] 1}+2 L_{1[j} \Psi_{k] i}, \\
0 & =\Psi_{i\{k} A_{j m\}} .
\end{aligned}
$$

Let us first show that from equation (26) it follows that the multiple PND $\ell$ in type $\mathrm{N}$ vacuum spacetimes is geodesic. For simplicity we will denote $L_{i 0}$ as $L_{i}$.

The contraction of $i$ with $k$ in (26) leads to

$$
\Psi_{i j} L_{i}=0 .
$$

Now the contraction of (26) with $\Psi_{i k}$ gives

$$
\Psi_{i k} \Psi_{i k} L_{j}=0 .
$$

Note that in type $\mathrm{N}$ spacetimes $\Psi_{i k} \Psi_{i k}>0$ and thus the previous equation implies $L_{i}=0$ and $\boldsymbol{\ell}$ is indeed geodesic.

By substituting $\boldsymbol{L}=\boldsymbol{A}+\boldsymbol{S}$ into (27) and using (28), we obtain

$$
S_{k[j} \Psi_{m] i}+S_{i[m} \Psi_{j] k}=0 .
$$

Let us now study in detail consequences of equations (25), (28) and (29). It is possible to find a general solution of these equations by taking into account their various contractions (hereafter we assume $\boldsymbol{S} \neq 0$ and $\boldsymbol{A} \neq 0$; the non-twisting case can be obtained as a special case with $\boldsymbol{A}=0$ ).

Contracting $i$ with $j$ in (28) gives

$$
\Psi_{i k} A_{i m}=\Psi_{i m} A_{i k} \quad \Rightarrow \quad \boldsymbol{\Psi} \cdot \boldsymbol{A}+\boldsymbol{A} \cdot \boldsymbol{\Psi}=0
$$

and contracting $k$ with $j$ in (29) leads to

$$
\Psi_{i j} S_{j m}+\Psi_{j m} S_{i j}=2 S \Psi_{i m} \quad \Rightarrow \quad \boldsymbol{\Psi} \cdot \boldsymbol{S}+\boldsymbol{S} \cdot \boldsymbol{\Psi}=2 S \boldsymbol{\Psi} .
$$

Previous two equations imply

$$
\boldsymbol{\Psi} \cdot \boldsymbol{L}+\boldsymbol{L} \cdot \boldsymbol{\Psi}=2 S \boldsymbol{\Psi} .
$$

By contracting $i$ with $m$ in (31) we get

$$
\Psi_{i j} S_{i j}=0 \Rightarrow \Psi_{i j} L_{i j}=0 .
$$


From (25) and (30) it follows that

$$
\Psi_{k i} S_{k j}=\Psi_{k j} S_{k i} \Rightarrow \boldsymbol{\Psi} \cdot \boldsymbol{S}-\boldsymbol{S} \cdot \boldsymbol{\Psi}=0 .
$$

Let us denote a trace $[\Psi \cdot \Psi]$ as $p$. Now we are in a position to formulate the following lemma.

Lemma 1 In vacuum type $N$ spacetimes with $\boldsymbol{L} \neq 0$ following relations are satisfied:

$$
\begin{aligned}
& \text { (a) } \boldsymbol{\Psi} \cdot \boldsymbol{S}=S \boldsymbol{\Psi}, \\
& \text { (b) } \boldsymbol{S} \cdot \boldsymbol{S}=S \boldsymbol{S}, \\
& \text { (c) } \boldsymbol{A} \cdot \boldsymbol{S}=\boldsymbol{S} \cdot \boldsymbol{A}=S \boldsymbol{A}, \\
& \text { (d) } S \boldsymbol{A} \cdot \boldsymbol{A}=-A^{2} \boldsymbol{S}, \\
& \text { (e) } 2 S \boldsymbol{\Psi} \cdot \boldsymbol{\Psi}=p \boldsymbol{S}, \\
& \text { (f) } S \boldsymbol{L} \cdot \boldsymbol{L}^{T}=S \boldsymbol{L}^{T} \cdot \boldsymbol{L}=\left(S^{2}+A^{2}\right) \boldsymbol{S} .
\end{aligned}
$$

Proof. (a) This equation is a direct consequence of (31) and (33).

(b) By multiplying (29) by $S_{i p}$ and using Lemma 1(a) we get

$$
\Psi_{k j} S_{i m} S_{i p}+S \Psi_{m p} S_{k j}=\Psi_{k m} S_{i j} S_{i p}+S \Psi_{j p} S_{k m} .
$$

An appropriate linear combination of this equation with (29) leads to

$$
\Psi_{k j}\left(S_{i m} S_{i p}-S S_{m p}\right)=\Psi_{k m}\left(S_{i j} S_{i p}-S S_{p j}\right),
$$

which, denoting $X_{m p} \equiv S_{i m} S_{i p}-S S_{m p}$, takes the form

$$
\Psi_{k j} X_{m p}=\Psi_{k m} X_{j p}
$$

Note that the matrix $\boldsymbol{X}$ is symmetric. Now contracting $k$ with $j$ gives

$$
\Psi_{j m} X_{j p}=0 .
$$

Multiplying (40) with $X_{m r}$ and using (41) leads to $X_{m p} X_{m r}=0$, which, contracting $p$ with $r$, gives $X_{m p} X_{m p}=0$ and consequently $X_{m p}=0$.

(c) Multiplying (28) with $S_{k p}$ and using Lemma 1(a) leads to

$$
\psi_{i j} A_{k m} S_{k p}+S \psi_{i p} A_{m j}+\psi_{i m} A_{j k} S_{k p}=0 .
$$

Now contracting $i$ with $m$ and using (30) gives

$$
\psi_{i j} Y_{i p}=0
$$

where $Y_{i p} \equiv A_{k i} S_{k p}+S A_{i p}$. Now substituting $A_{k i} S_{k p}=Y_{i p}-S A_{i p}$ into (42) and using (28) leads to

$$
\psi_{i j} Y_{m p}=\psi_{i m} Y_{j p}
$$

Multiplying (44) with $Y_{m r}$ using (43) gives $Y_{m p} Y_{m r}=0$ and consequently $Y_{m p}=0$. (d) Let us define another symmetric matrix $\boldsymbol{B}$ by $B_{i k} \equiv A_{i j} A_{j k}$. Note that $[\boldsymbol{B}]=$ $-2 A^{2}$. Multiplying (30) by $A_{m r}$ and again applying (30) leads to

$$
\psi_{i k} B_{i r}=\psi_{i r} B_{i k} .
$$

Multiplying (28) by $A_{m r}$ leads to

$$
\psi_{i j} B_{k r}-\psi_{i k} B_{j r}+\psi_{i m} A_{j k} A_{m r}=0,
$$

which after contracting $k$ with $r$ gives

$$
\psi_{i k} B_{j k}=-A^{2} \Psi_{i j} .
$$


Multiplying (46) with $B_{k r}$ and using (45), (47) and (30) leads to $B_{k r} B_{k r}=2 A^{4}$ and similarly multiplying (46) by $S_{k r}$ results in $B_{k r} S_{k r}=-2 S A^{2}$. Let us now define a symmetric matrix $\boldsymbol{Q}$ by

$$
Q_{i j} \equiv S B_{i j}+A^{2} S_{i j}
$$

Using previous formulae it turns out that $Q_{i j} Q_{i j}=0$ and thus $Q_{i j}=0$.

(e) This follows from multiplying (29) by $\Psi_{k j}$.

(f) This follows directly from Lemma 1(b)-(d).

Let us now use Lemma 1 to prove following lemmas for vacuum type $\mathrm{N}$ spacetimes with $\boldsymbol{L} \neq 0$ :

Lemma 2 The matrix $\boldsymbol{S}$ has at most two eigenvalues $\lambda=0$ and $\lambda=S$.

Proof. Let us denote the eigenvector of $\boldsymbol{S}$ by $\xi$. We thus have $S_{i j} \xi_{j}=\lambda \xi_{i}$. By multiplying Lemma 1(b) by $\xi$ we obtain

$$
S_{i j} S_{j k} \xi_{k}=S S_{i k} \xi_{k} \quad \Rightarrow \quad \lambda^{2}=S \lambda
$$

and thus $\lambda=0$ or $\lambda=S$.

Lemma 3 The following statements are equivalent:

(a) Vector $\xi$ is an eigenvector of $\boldsymbol{S}$ for the eigenvalue $\lambda=0$.

(b) Vector $\xi$ is an eigenvector of $\boldsymbol{A}$ for the eigenvalue $\lambda=0$.

(c) Vector $\xi$ is an eigenvector of $\boldsymbol{\Psi}$ for the eigenvalue $\lambda=0$.

Proof. (a) $\Rightarrow$ (b): Suppose that $S_{i j} \xi_{j}=0$. Then by multiplying Lemma 1(c) in the form $S_{i j} A_{j k}=S A_{i k}$ by $\xi_{i}$ we obtain $A_{i k} \xi_{i}=0$.

(b) $\Rightarrow$ (a): Suppose that $A_{i j} \xi_{j}=0$. Then by multiplying Lemma 1(d) in the form $S A_{i j} A_{j k}=-A^{2} S_{i k}$ by $\xi_{k}$ we obtain $S_{i k} \xi_{k}=0$.

(a) $\Rightarrow$ (c): Suppose that $S_{i j} \xi_{j}=0$. Then by multiplying Lemma 1(a) in the form $\Psi_{i j} S_{j k}=S \Psi_{i k}$ by $\xi_{k}$ we obtain $\Psi_{i k} \xi_{k}=0$.

(c) $\Rightarrow$ (a): Suppose that $\Psi_{i j} \xi_{j}=0$. Then by multiplying Lemma 1(e) in the form $2 S \Psi_{i j} \Psi_{j k}=p S_{i k}$ by $\xi_{k}$ we obtain $S_{i k} \xi_{k}=0$.

Lemma 4 Every eigenvector of $\boldsymbol{\Psi}$ is also an eigenvector of $\boldsymbol{S}$.

Proof. The case $\lambda=0$ is solved in Lemma 3. Let us now suppose that $\Psi_{i j} \xi_{j}=\lambda \xi_{i}$, $\lambda \neq 0$. Then by multiplying Lemma 1(a) in the form $\Psi_{i j} S_{j k}=S \Psi_{i k}$ by $\xi_{i}$ we obtain $S_{j k} \xi_{j}=S \xi_{k}$.

Lemma 5 The only possible eigenvalues of $\boldsymbol{\Psi}$ are $\lambda=0$ and $\lambda= \pm \sqrt{p / 2}$.

Proof. Let us now suppose that $\Psi_{i j} \xi_{j}=\lambda \xi_{i}, \lambda \neq 0$. Than thanks to Lemma 4 $S_{i j} \xi_{j}=S \xi_{i}$. By multiplying Lemma $1(\mathrm{e})$ in the form $2 S \Psi_{i j} \Psi_{j k}=p S_{i k}$ by $\xi_{k}$ we obtain $\lambda^{2}=p / 2$.

For every symmetric matrix $\boldsymbol{W}$ there exists an orthonormal basis of its eigenvectors $\xi_{i}^{a}$ in which

$$
W_{i j}=\sum_{a} \lambda_{a} \xi_{i}^{a} \xi_{j}^{a}
$$


Let us denote eigenvectors of $\boldsymbol{\Psi}$ corresponding to $\lambda=0, \lambda=\sqrt{p / 2}$ and $\lambda=-\sqrt{p / 2}$ by $\boldsymbol{u}^{\alpha}, \boldsymbol{v}^{A}$ and $\boldsymbol{w}^{\tilde{A}}$, respectively. Here indices $\alpha, \beta, \ldots, A, B, \ldots$ and $\tilde{A}, \tilde{B}, \ldots$ distinguish between vectors $\boldsymbol{u}^{1}, \ldots, \boldsymbol{u}^{\alpha_{\max }}, \boldsymbol{v}^{1}, \ldots, \boldsymbol{v}^{A_{\max }}$ and $\boldsymbol{w}^{1}, \ldots, \boldsymbol{w}^{\tilde{A}_{\max }}$. Now we have

$$
\Psi_{i j}=\sqrt{\frac{p}{2}}\left(v_{i}^{A} v_{j}^{A}-w_{i}^{\tilde{A}} w_{j}^{\tilde{A}}\right) .
$$

Note that for indices $\alpha, \beta, \ldots, A, B, \ldots$ and $\tilde{A}, \tilde{B}, \ldots$ we also observe Einstein's summation convention. Thanks to Lemmas 3 and 4

$$
S_{i j}=S\left(v_{i}^{A} v_{j}^{A}+w_{i}^{\tilde{A}} w_{j}^{\tilde{A}}\right) .
$$

Let us now multiply (29) by $v_{k}^{A} v_{j}^{B}$. This leads to

$$
\delta^{A B}\left(\sqrt{\frac{p}{2}} S_{i l}+S \Psi_{i l}\right)=S \sqrt{2 p} v_{l}^{A} v_{i}^{B}
$$

which for $A \neq B$ gives $v_{l}^{A} v_{i}^{B}=0$ and thus just one value of index $A$ is possible and consequently $A_{\max }=1$. Let us thus denote $\boldsymbol{v}^{1}$ by $\boldsymbol{v}$. Similarly, we can find that $\tilde{A}_{\max }=1$ and that there is thus just one vector $\boldsymbol{w}=\boldsymbol{w}^{1}$. Equations (48) and (49) now take form

$$
\Psi_{i j}=\sqrt{\frac{p}{2}}\left(v_{i} v_{j}-w_{i} w_{j}\right), \quad S_{i j}=S\left(v_{i} v_{j}+w_{i} w_{j}\right) .
$$

Let us now introduce a new vector $\boldsymbol{V}$ by

$$
V_{i}=A_{i j} v_{j}
$$

Thanks to Lemma $1(\mathrm{c})$ and (d) we have

$$
S_{i j} V_{i}=S_{i j} A_{i k} v_{k}=S V_{j}, \quad V_{i} v_{i}=0, \quad V_{i} V_{i}=A^{2}
$$

and thus $\boldsymbol{V}$ is just a multiple of $\boldsymbol{w}\left(w_{i}= \pm \frac{1}{A} V_{i}\right)$ and we have freedom to set $w_{i}=\frac{1}{A} V_{i}$. Then we have

$$
A_{i j} v_{j}=A w_{i}, \quad A_{i j} w_{j}=-A v_{i} .
$$

Now by multiplying equation (28) by $v_{i} v_{j}$ we get

$$
A_{k l}=A\left(w_{k} v_{l}-v_{k} w_{l}\right) \text {. }
$$

Note that $\boldsymbol{\Psi}, \boldsymbol{S}$ and $\boldsymbol{A}$ given by (50) and (51) satisfy equations (28), (29) and thus represent the general solution of these equations for $\boldsymbol{L} \neq 0$.

\section{Type III vacuum spacetimes}

For type III vacuum spacetimes the Weyl tensor is given by (11), where $\Psi_{i j k}$ satisfies (12) and (13). The algebraic equations

$$
\begin{aligned}
& \Psi_{i j k} L_{k}=L_{[i} \Psi_{j]}, \\
& \Psi_{k l[j} L_{i]}=\Psi_{i j[k} L_{l]}, \\
& L_{i[j} \Psi_{k]}+2 L_{s[j \mid} \Psi_{s i \mid k]}=0, \\
& 2 A_{\{i j \mid} \Psi_{k l \mid m\}}+L_{l\{i} \Psi_{j m\} k}-L_{k\{i} \Psi_{j m\} l}=0,
\end{aligned}
$$

where we denote $L_{i 0}$ as $L_{i}$, follow from the Bianchi equations given in Appendix B. 
Let us show that from (52) and (53) it follows that the multiple PND $\ell$ in vacuum type III spacetimes is geodesic. By contracting $l$ with $j$ in (53) and using (52), (12), and (13) we obtain

$$
2 \Psi_{i j k} L_{j}=\Psi_{k} L_{i} .
$$

One can see that for the class with $\Psi_{k} \neq 0$ it implies (as we can see by contracting this formula with $L_{i}$ ) that $L_{i}=0$. Similarly, for the case $\Psi_{k}=0$ these equations also imply that $L_{i}=0$ (contract (53) with $L_{i}$ and employ (52) and (56)). Thus, the multiple PND $\ell$ in vacuum type III spacetimes is geodesic.

Now the task is to solve (54) and (55). This turns out to be quite complicated. We will be able to find a general solution in an arbitrary dimension in the non-twisting case with $A_{i j}=0$ (see Appendix C.1). We also present a solution for the twisting case, but we can prove that it is a general solution only if we assume that $\Psi_{i j k}$ is in a 'general form'. In Appendix C.2, we show that this solution is indeed general in all degenerate cases in five dimensions.

Let us start with extracting some information from (54) and (55) by various contractions. By contracting (55) and using (54) we get

$$
L \Psi_{i j k}+2 L_{[i \mid s} \Psi_{s k \mid j]}-2 S_{s k} \Psi_{i j s}+L_{[i \mid k} \Psi_{\mid j]}=0,
$$

where $L=2 S$ is the trace of $\boldsymbol{L}$. After adding (54) (where we replace indices $i, j, k$ by $k, i, j$, respectively) to (57) we obtain

$$
L \Psi_{i j k}+4 S_{[i \mid s} \Psi_{s k \mid j]}-2 S_{s k} \Psi_{i j s}+2 S_{[i \mid k} \Psi_{\mid j]}=0,
$$

which does not contain $\boldsymbol{A}$ - the antisymmetric part of $\boldsymbol{L}$, and similarly

$$
L \Psi_{i j k}-4 \Psi_{s k[i} A_{j] s}-2 \Psi_{[i} A_{j] k}-2 \Psi_{i j s} S_{s k}=0 .
$$

Contraction of $i$ with $j$ in (54) leads to

$$
L \Psi_{k}-2 L_{i k} \Psi_{i}+2 L_{s i} \Psi_{s i k}=0
$$

and contraction of $k$ with $j$ in (58) (using (12) and (13)) to

$$
L_{i j} \Psi_{j}=2 L_{j s} \Psi_{i j s}
$$

and (60), using (13), with (61) imply

$$
L \Psi_{i}-L_{i j} \Psi_{j}-2 L_{j i} \Psi_{j}+2 L_{k j} \Psi_{i j k}=0 .
$$

By substituting $\boldsymbol{L}=\boldsymbol{S}+\boldsymbol{A}$ into (60) and (62) we get

$$
\begin{aligned}
& L \Psi_{i}-2 S_{i j} \Psi_{j}-2 A_{j i} \Psi_{j}-4 A_{j k} \Psi_{i j k}=0, \\
& L \Psi_{i}-3 S_{i j} \Psi_{j}-A_{j i} \Psi_{j}+2\left(S_{j k}-A_{j k}\right) \Psi_{i j k}=0
\end{aligned}
$$

and their linear combinations give

$$
\begin{aligned}
& L \Psi_{i}+4 A_{i j} \Psi_{j}-4\left(S_{j k}+2 A_{j k}\right) \Psi_{i j k}=0, \\
& L \Psi_{i}-4 S_{i j} \Psi_{j}+4 S_{j k} \Psi_{i j k}=0 .
\end{aligned}
$$

By multiplying (54) by $L_{i j}$ we arrive to

$$
L_{i j} L_{i k} \Psi_{j}=\frac{1}{2} \ell \Psi_{k},
$$

where $\ell=L_{i j} L_{i j}$.

Inspired by the type $\mathrm{N}$ case, we again choose an orthonormal basis of eigenvectors of $\boldsymbol{S}$. We will denote vectors corresponding to non-zero eigenvalues of $\boldsymbol{S}$ as 
$\boldsymbol{v}^{1}, \boldsymbol{v}^{2}, \ldots, \boldsymbol{v}^{A_{\max }}$ and vectors corresponding to the eigenvalue 0 as $\boldsymbol{u}^{1}, \boldsymbol{u}^{2}, \ldots, \boldsymbol{u}^{\alpha_{\max }}$. We thus have

$$
S_{i j}=\sum_{A=1}^{A_{\max }} \lambda_{A} v_{i}^{A} v_{j}^{A} .
$$

Unfortunately, there are three indices $A$ in this formula and we thus will not use Einstein's summation convention for indices $A, B, \ldots$ (though we will still use it for indices $\alpha, \beta, \ldots)$.

Let us now decompose other quantities appearing in (54) and (55)

$$
\begin{aligned}
\Psi_{i}= & \sum_{A} a_{A} v_{i}^{A}+b_{\alpha} u_{i}^{\alpha}, \\
A_{i j}= & \sum_{A B} \mathcal{A}_{A B}\left(v_{i}^{A} v_{j}^{B}-v_{j}^{A} v_{i}^{B}\right)+\sum_{A} \mathcal{B}_{A \beta}\left(v_{i}^{A} u_{j}^{\beta}-v_{j}^{A} u_{i}^{\beta}\right)+\mathcal{C}_{\alpha \beta}\left(u_{i}^{\alpha} u_{j}^{\beta}-u_{j}^{\alpha} u_{i}^{\beta}\right), \\
\Psi_{i j k}= & \sum_{A B C} \mathcal{M}_{A B C}\left(v_{i}^{A} v_{j}^{B}-v_{j}^{A} v_{i}^{B}\right) v_{k}^{C}+\sum_{A B} \mathcal{N}_{A B \gamma}\left(v_{i}^{A} v_{j}^{B}-v_{j}^{A} v_{i}^{B}\right) u_{k}^{\gamma} \\
& +\sum_{A C} \mathcal{O}_{A \beta C}\left(v_{i}^{A} u_{j}^{\beta}-v_{j}^{A} u_{i}^{\beta}\right) v_{k}^{C}+\sum_{A} \mathcal{P}_{A \beta \gamma}\left(v_{i}^{A} u_{j}^{\beta}-v_{j}^{A} u_{i}^{\beta}\right) u_{k}^{\gamma} \\
& +\sum_{C} \mathcal{R}_{\alpha \beta C}\left(u_{i}^{\alpha} u_{j}^{\beta}-u_{j}^{\alpha} u_{i}^{\beta}\right) v_{k}^{C}+\mathcal{S}_{\alpha \beta \gamma}\left(u_{i}^{\alpha} u_{j}^{\beta}-u_{j}^{\alpha} u_{i}^{\beta}\right) u_{k}^{\gamma},
\end{aligned}
$$

where $\mathcal{A}, \mathcal{C}, \mathcal{M}, \mathcal{N}, \mathcal{R}$ and $\mathcal{S}$ are antisymmetric in first two indices. Now we need to rewrite some of the equations from this section in terms of these new quantities (more complicated equations are given in Appendix A.

From (12) and (66), it follows that

$$
\begin{aligned}
& a_{A}=4 \sum_{B} \mathcal{M}_{A B B}+2 \mathcal{P}_{A \beta \beta}, \\
& b_{\alpha}=-2 \sum_{B} \mathcal{O}_{B \alpha B}+4 \mathcal{S}_{\alpha \beta \beta},
\end{aligned}
$$

and

$$
\begin{aligned}
\left(L-4 \lambda_{A}\right) a_{A} & =-8 \sum_{B} \lambda_{B} \mathcal{M}_{A B B}, \\
L b_{\alpha} & =4 \sum_{B} \lambda_{B} \mathcal{O}_{B \alpha B},
\end{aligned}
$$

respectively. Equation (58) implies

$$
\begin{aligned}
& L \mathcal{S}_{\alpha \beta \gamma}=0 \\
& \left(L-2 \lambda_{C}\right) \mathcal{R}_{\alpha \beta C}=0 \\
& L \mathcal{P}_{B \alpha \gamma}+2 \lambda_{B} \mathcal{P}_{B \gamma \alpha}=0 \\
& \left(L-2 \lambda_{C}\right) \mathcal{O}_{A \beta C}+4 \lambda_{A} \mathcal{N}_{A C \beta}+\lambda_{A} b_{\beta} \delta_{A C}=0 \\
& 2 \lambda_{A} \mathcal{O}_{A \gamma B}-2 \lambda_{B} \mathcal{O}_{B \gamma A}+2 L \mathcal{N}_{A B \gamma}=0 \\
& \left(L-2 \lambda_{C}\right) \mathcal{M}_{A B C}+2 \lambda_{A} \mathcal{M}_{A C B}+2 \lambda_{B} \mathcal{M}_{C B A}+\lambda_{C} \delta_{C[A} a_{B]}=0
\end{aligned}
$$

and (13) leads to

$$
\begin{aligned}
& \mathcal{P}_{A[\beta \gamma]}+\mathcal{R}_{\beta \gamma A}=0, \\
& \mathcal{O}_{[A|\beta| C]}+\mathcal{N}_{C A \beta}=0, \\
& \mathcal{M}_{\{A B C\}}=0, \\
& \mathcal{S}_{\{A B C\}}=0 .
\end{aligned}
$$


In the twisting case, the equations (54) and (55), without specifying dimension, are quite complex, and there are too many distinct cases to be solved. We thus present the solution provided $L \neq 0$ and provided that for every pair $A, C(A \neq C)$ there exists $\beta$ for which $\mathcal{O}_{A \beta C} \neq 0$. It turns out that the only eigenvalues of $\boldsymbol{S}$ that are compatible with (54) and (55) correspond to $A_{\max }=2, \lambda_{1}=L / 2, \lambda_{2}=L / 2$. We also checked other cases (but not all of them) and they also lead to the same conclusion. For completeness, we treat all other possible cases in five dimensions in Appendix C.2.

A linear combination of (78) and (81), [(178) - $L$ (81)], gives

$$
\left(2 \lambda_{A}+L\right) \mathcal{O}_{A \gamma B}=\left(2 \lambda_{B}+L\right) \mathcal{O}_{B \gamma A} .
$$

For $A \neq B\left[\right.$ [(77) $-2 \lambda_{A}$ (81)] leads to

$$
\left(L+2 \lambda_{A}-2 \lambda_{B}\right) \mathcal{O}_{A \gamma B}=\left.2 \lambda_{A} \mathcal{O}_{B \gamma A}\right|_{A \neq B},
$$

and $\left[2 \lambda_{A}\right.$ (84) $-\left(2 \lambda_{B}+L\right)$ (85) $]$ gives

$$
\left(\lambda_{A}^{2}+\lambda_{B}^{2}-\lambda_{A} \lambda_{B}-\frac{L^{2}}{4}\right) \mathcal{O}_{A \gamma B}=\left.0\right|_{A \neq B} .
$$

If $\mathcal{O}_{A \gamma B} \neq 0$, we obtain from (86)

$$
\left(\lambda_{A}^{2}+\lambda_{B}^{2}-\lambda_{A} \lambda_{B}-\frac{L^{2}}{4}\right)=\left.0\right|_{A \neq B} .
$$

If for each pair $A, B$ there exists $\gamma$ such that $\mathcal{O}_{A \gamma B} \neq 0$ then (87) is valid for all $A$, $B, A \neq B$. In the case $A_{\max }=2$, we have $L=\lambda_{1}+\lambda_{2}$ and equation (86) leads to $\left(\lambda_{1}-\lambda_{2}\right)^{2}=0$ and consequently $\lambda_{1}=\lambda_{2}=\frac{L}{2}$.

In the case $A_{\max }>2$, by subtracting

$$
\left(\lambda_{A}^{2}+\lambda_{C}^{2}-\lambda_{A} \lambda_{C}-\frac{L^{2}}{4}\right)=\left.0\right|_{A \neq C}
$$

from (87) we obtain

$$
\left(\lambda_{B}-\lambda_{C}\right)\left(\lambda_{B}+\lambda_{C}-\lambda_{A}\right)=\left.0\right|_{A \neq B, A \neq C, B \neq C}
$$

and thus

$$
\lambda_{B}-\lambda_{C}=0
$$

or

$$
\lambda_{B}+\lambda_{C}-\left.\lambda_{A}\right|_{A \neq B, A \neq C, B \neq C}=0 .
$$

However, in the second case one can show that simply by summing this equation with interchanged indices

$$
\left(\lambda_{B}+\lambda_{C}-\lambda_{A}=0\right)+\left(\lambda_{B}+\lambda_{A}-\lambda_{C}=0\right) \Rightarrow \lambda_{A}=0
$$

which cannot happen as $\lambda_{A} \neq 0$ by definition and thus (89) is satisfied and by substituting $\lambda_{A}=\lambda_{B}=\lambda$ in (87) we obtain $\lambda= \pm \frac{L}{2}$. Note however that $\sum_{A} \lambda_{A}=L$ and thus the case $\lambda_{A}=-L / 2$ is excluded and $A_{\max }=2$. Thus we can conclude with

Lemma 6 For vacuum type III spacetimes with $L \neq 0$, providing that for every pair $A, C(A \neq C)$ there exists $\beta$ for which $\mathcal{O}_{A \beta C} \neq 0$, the expansion matrix $\boldsymbol{S}$ has just one non-vanishing eigenvalue $S=\frac{L}{2}$ which has multiplicity 2.

Let us now study the case with two non-vanishing eigenvalues of $\boldsymbol{S}, \lambda_{1}=\lambda_{2}=$ $L / 2$, in general, i.e. without any assumptions about $\mathcal{O}_{A \beta C}$. 
4.1. The case $A_{\max }=2, \lambda_{1}=\lambda_{2}=L / 2$ and $\Psi_{i} \neq 0$.

From (74) and (76) it follows that

$$
\mathcal{S}_{\alpha \beta \gamma}=0, \quad \mathcal{P}_{A \beta \gamma}=-\mathcal{P}_{A \gamma \beta} .
$$

By assuming that the index $A \neq C$ in (77) we obtain

$$
\mathcal{N}_{A C \beta}=0 \text {. }
$$

Since $\mathcal{N}$ is antisymmetric in the first two indices, $\mathcal{N}_{A C \beta}=0$ for all combinations of indices. By substituting $A=C$ in (77) we obtain

$$
b_{\beta}=0
$$

and thus $\Psi_{i}$ is an eigenvector of $\boldsymbol{S}$ corresponding to $L / 2$. Equation (77) now implies

$$
\mathcal{O}_{A \beta C}=\mathcal{O}_{C \beta A}
$$

and (71) implies

$$
\sum_{A} \mathcal{O}_{A \beta A}=0
$$

Substituting $A \neq C, B \neq C$ into (79) leads to

$$
\mathcal{M}_{A B C}=\left.0\right|_{A \neq C, B \neq C},
$$

(70) leads to

$$
a_{A}=4 \sum_{B} \mathcal{M}_{A B B} \quad \Rightarrow \quad a_{1}=4 \mathcal{M}_{122}, a_{2}=4 \mathcal{M}_{211}
$$

and (80) gives

$$
\mathcal{P}_{A \beta \gamma}+\mathcal{R}_{\beta \gamma A}=0 .
$$

Substituting the index $B=A$ into A.5), summing over the index $A$ and using (A.1), (92), (91) and (90) we arrive at $\sum_{A} \mathcal{B}_{A \beta} a_{A}=0$. Consequently, the vector $\boldsymbol{\Phi}$

$$
\Phi_{i}=A_{i j} \Psi_{j}=\sum_{B} 2 \mathcal{A}_{A B} a_{B} v_{i}^{A},
$$

which is orthogonal to $\Psi_{i}$, is also an eigenvector of $\boldsymbol{S}$. We thus have two orthogonal eigenvectors of $\boldsymbol{S}, \Psi_{i}$ and $\Phi_{i}$. Let us denote $\Psi_{i} \Psi_{i}$ by $\psi^{2}$ and $\Phi_{i} \Phi_{i}$ by $\phi^{2}$. Now we choose an orthogonal basis with these two vectors which corresponds to

$$
v_{i}^{1}, v_{i}^{2} \longmapsto \frac{\Psi_{i}}{\psi}, \frac{\Phi_{i}}{\phi}
$$

In this basis we will denote components corresponding to $\Psi_{i}$ and $\Phi_{i}$ by indices $P$ and $F$, respectively. $\boldsymbol{S}$ now takes the form

$$
S_{i j}=S\left(\frac{\Psi_{i} \Psi_{j}}{\psi^{2}}+\frac{\Phi_{i} \Phi_{j}}{\phi^{2}}\right) .
$$

This equation implies $S_{i j} S_{i j}=2 S^{2}$. Now using $\ell=2\left(A^{2}+S^{2}\right)$ it follows from the previous equations and (67) that

$$
A_{i k} \Phi_{i}=A^{2} \Psi_{k}
$$

and thus

$$
\phi^{2}=\Phi_{i} \Phi_{i}=\Phi_{i} A_{i k} \Psi_{k}=A^{2} \Psi_{k} \Psi_{k}=A^{2} \psi^{2}
$$


$A_{i j}$ as an antisymmetric matrix takes the general form

$$
\begin{aligned}
A_{i j}= & \mathcal{A}_{P F}\left(\Psi_{i} \Phi_{j}-\Psi_{j} \Phi_{i}\right)+\mathcal{B}_{P \alpha}\left(\Psi_{i} u_{j}^{\alpha}-\Psi_{j} u_{i}^{\alpha}\right) \\
+ & \mathcal{B}_{F \alpha}\left(\Phi_{i} u_{j}^{\alpha}-\Phi_{j} u_{i}^{\alpha}\right)+\mathcal{C}_{\alpha \beta}\left(u_{i}^{\alpha} u_{j}^{\beta}-u_{j}^{\alpha} u_{i}^{\beta}\right)
\end{aligned}
$$

but from $A_{i j} \Psi_{j}=\Phi_{i}$ and $A_{i k} \Phi_{i}=A^{2} \Psi_{k}$ we get

$$
\mathcal{B}_{P \alpha}=0, \quad \mathcal{A}_{P F}=-\frac{1}{\psi^{2}}, \quad \mathcal{B}_{F \alpha}=0
$$

and thus

$$
A_{i j}=\frac{1}{\psi^{2}}\left(\Phi_{i} \Psi_{j}-\Phi_{j} \Psi_{i}\right)+\mathcal{C}_{\alpha \beta}\left(u_{i}^{\alpha} u_{j}^{\beta}-u_{j}^{\alpha} u_{i}^{\beta}\right) .
$$

Thanks to (90) - 93) we can rewrite $\Psi_{i j k}$ in the form

$$
\begin{aligned}
& \Psi_{i j k}=\frac{1}{2 \phi^{2}}\left(\Psi_{i} \Phi_{j}-\Psi_{j} \Phi_{i}\right) \Phi_{k}+\frac{\mathcal{O}_{P \alpha P}}{\psi^{2}}\left(\Psi_{i} u_{j}^{\alpha}-\Psi_{j} u_{i}^{\alpha}\right) \Psi_{k}-\frac{\mathcal{O}_{P \alpha P}}{\phi^{2}}\left(\Phi_{i} u_{j}^{\alpha}-\Phi_{j} u_{i}^{\alpha}\right) \Phi_{k} \\
& +\frac{\mathcal{O}_{P \alpha F}}{\psi \phi}\left(\Psi_{i} u_{j}^{\alpha}-\Psi_{j} u_{i}^{\alpha}\right) \Phi_{k}+\frac{\mathcal{O}_{P \alpha F}}{\psi \phi}\left(\Phi_{i} u_{j}^{\alpha}-\Phi_{j} u_{i}^{\alpha}\right) \Psi_{k}+\frac{\mathcal{P}_{P \beta \gamma}}{\psi}\left(\Psi_{i} u_{j}^{\beta}-\Psi_{j} u_{i}^{\beta}\right) u_{k}^{\gamma} \\
& +\frac{\mathcal{P}_{F \beta \gamma}}{\phi}\left(\Phi_{i} u_{j}^{\beta}-\Phi_{j} u_{i}^{\beta}\right) u_{k}^{\gamma}-\frac{\mathcal{P}_{P \beta \gamma}}{\psi}\left(u_{i}^{\beta} u_{j}^{\gamma}-u_{j}^{\beta} u_{i}^{\gamma}\right) \Psi_{k}-\frac{\mathcal{P}_{F \beta \gamma}}{\phi}\left(u_{i}^{\beta} u_{j}^{\gamma}-u_{j}^{\beta} u_{i}^{\gamma}\right) \Phi_{k} .
\end{aligned}
$$

By substituting (95) into (A.15) we obtain $\mathcal{A}_{P F} \mathcal{P}_{P \gamma \delta}=0, \mathcal{A}_{P F} \mathcal{P}_{F \gamma \delta}=0$ and since $\mathcal{A}_{P F} \neq 0$ we also get $\mathcal{P}_{P \gamma \delta}=0=\mathcal{P}_{F \gamma \delta}$. Then A.14 or A.13) gives $\mathcal{C}_{\alpha \beta} \mathcal{M}_{C D E}=0$ which, since $\mathcal{M}_{C D E}$ does not vanish, implies $\mathcal{C}_{\alpha \beta}=0$. In this case we thus have

$$
A_{i j}=\frac{1}{\psi^{2}}\left(\Phi_{i} \Psi_{j}-\Phi_{j} \Psi_{i}\right)
$$

and

$$
\begin{aligned}
\Psi_{i j k} & =\frac{1}{2 \phi^{2}}\left(\Psi_{i} \Phi_{j}-\Psi_{j} \Phi_{i}\right) \Phi_{k} \\
& +\frac{\mathcal{O}_{P \alpha P}}{\psi^{2}}\left(\Psi_{i} u_{j}^{\alpha}-\Psi_{j} u_{i}^{\alpha}\right) \Psi_{k}-\frac{\mathcal{O}_{P \alpha P}}{\phi^{2}}\left(\Phi_{i} u_{j}^{\alpha}-\Phi_{j} u_{i}^{\alpha}\right) \Phi_{k} \\
& +\frac{\mathcal{O}_{P \alpha F}}{\psi \phi}\left(\Psi_{i} u_{j}^{\alpha}-\Psi_{j} u_{i}^{\alpha}\right) \Phi_{k}+\frac{\mathcal{O}_{P \alpha F}}{\psi \phi}\left(\Phi_{i} u_{j}^{\alpha}-\Phi_{j} u_{i}^{\alpha}\right) \Psi_{k} .
\end{aligned}
$$

We do not need to examine the rest of equations in Appendix A since $S_{i j}, A_{i j}$ and $\Psi_{i j k}$ given by (94), (96) and (97), respectively, already satisfy both equations (54) and (55) and thus represent their solution.

Note that from (94), (96) and (97) we obtain for type III spacetimes relations equal or analogous to equations given in Lemma 1;

$$
\begin{aligned}
& \text { (a) } \Psi_{i j s} S_{s k}=S \Psi_{i j k} \\
& \text { (b) } S_{i k} S_{j k}=S S_{i j} \\
& \text { (c) } A_{i k} S_{k j}=S_{i k} A_{k j}=S A_{i j} \\
& \text { (d) } S A_{i k} A_{k j}=-A^{2} S_{i j} \\
& \text { (e) } S L_{i k} L_{j k}=S L_{k i} L_{k j}=\left(S^{2}+A^{2}\right) S_{i j}
\end{aligned}
$$




\section{Comments on a possible generalization of the Goldberg-Sachs theorem for higher dimensions}

In Sections 3 and 4, it is shown that for type $\mathrm{N}$ and III vacuum spacetimes the expansion and twist matrices $\boldsymbol{S}$ and $\boldsymbol{A}$ have very specific properties given by (50), (51) and (94), (96) for type $\mathrm{N}$ and III, respectively. Note, however, that while in higher dimensions as well as in 4D the multiple PND is geodesic, it is not shearfree for $n>4$. The question thus arises whether there exist some properties of matrices $\boldsymbol{S}$ and $\boldsymbol{A}$ that are satisfied (together with the condition that the spacetime possesses a geodesic null congruence) if and only if the vacuum spacetime is algebraically special. The answer is unclear at present. Moreover, the conditions for $\boldsymbol{S}$ and $\boldsymbol{A}$ that hold for types $\mathrm{N}$ and III are not satisfied for type D spacetimes (see Appendix D). Let us here, as a first step towards such possible generalization, show that from the Ricci identities it follows that

Lemma 7 a) Suppose that an otherwise arbitrary vacuum spacetime admits a nonexpanding and non-twisting geodesic null congruence (i.e. $\boldsymbol{S}=0=\boldsymbol{A}$ ). Then, the spacetime in question is algebraically special.

b) Suppose that an otherwise arbitrary vacuum spacetime admits an expanding and twisting geodesic null congruence, and that its $\boldsymbol{S}$ and $\boldsymbol{A}$ matrices, in appropriately chosen frame, have the form (50), (51). Then, the spacetime in question is algebraically special.

Proof. The contraction of the Ricci identities $\ell_{a ; b c}-\ell_{a ; c b}-R_{a b c}^{s} \ell_{s}=0$ with $m^{(i) a} \ell^{b} m^{(j) c}$ assuming that $\boldsymbol{\ell}$ is geodesic with an affine parametrization leads to

$$
D L_{i j}+L_{i s} L_{s j}+L_{i s} \stackrel{s}{M}_{j 0}+L_{s j} \stackrel{s}{M}_{i 0}+R_{0 i 0 j}=0 .
$$

If $\boldsymbol{L}=\boldsymbol{S}+\boldsymbol{A}=0$ then $R_{0 i 0 j}=C_{0 i 0 j}=0$ for all $i, j$ and the spacetime is thus algebraically special. This proves the part (a) of Lemma 7

If we switch $i$ and $j$ in (103) and add and subtract the two equations we obtain

$$
\begin{aligned}
& 2 D S_{i j}+L_{i s} L_{s j}+L_{j s} L_{s i}+2 S_{i s} \stackrel{s}{M} j 0+2 S_{s j} \stackrel{s}{M} i 0+2 R_{0 i 0 j}=0, \\
& 2 D A_{i j}+L_{i s} L_{s j}-L_{j s} L_{s i}+2 A_{i s} \stackrel{s}{M} j 0+2 A_{s j} \stackrel{s}{M}=0 .
\end{aligned}
$$

We further assume that we have chosen vectors $\boldsymbol{m}^{(i)}$ in such a way that $\mathbf{S}$ is diagonal with the only non-zero entries being $S_{22}=S_{33}$ and that $\boldsymbol{A}$ has only two non-vanishing components $A_{23}=-A_{32}$. Then (104) takes the form

$$
2 D S_{i j}+\frac{2}{S}\left(S^{2}-A^{2}\right) S_{i j}+2 S_{i s} \stackrel{s}{M}_{j 0}+2 S_{s j} \stackrel{s}{M} i 0+2 R_{0 i 0 j}=0
$$

If $i, j>3$ then $R_{0 i 0 j}=0$. If $i \neq j<4$ then (106) implies $2 S_{22} \stackrel{2}{M_{30}}+2 S_{33} \stackrel{3}{M}_{20}+$ $2 R_{0203}=0$ which using (14) leads to $R_{0203}$. If $i=j<4$ then again from (106) we get $2 D S+2\left(S^{2}-A^{2}\right)+2 R_{0202}=2 D S+2\left(S^{2}-A^{2}\right)+2 R_{0303}=0$ which together with $R_{0 i 0 i}=0$ gives $R_{0202}=R_{0303}=0$. For $j>3$ and $i \in(2,3)$ equations (106) and (105) turn to be

$$
\begin{aligned}
& 2 S_{i s} \stackrel{s}{M}_{j 0}+2 R_{0 i 0 j}=0 \Longrightarrow S_{22} \stackrel{2}{M}_{j 0}+R_{020 j}=0, S_{33} \stackrel{3}{M}_{j 0}+R_{030 j}=0, \\
& 2 A_{i s} \stackrel{s}{M}{ }_{j 0}=0 \Longrightarrow A_{23} \stackrel{3}{M}{ }_{j 0}=A_{32} \stackrel{2}{M}{ }_{j 0}=0 \Longrightarrow \stackrel{2}{M}_{j 0}=\stackrel{3}{M}_{j 0}=0
\end{aligned}
$$

that leads to $R_{020 j}=R_{030 j}=0$ and hence $R_{0 i 0 j}=C_{0 i 0 j}=0$ for all $i, j$ and the spacetime is thus algebraically special. This proves the part (b) of Lemma 7 , 
Note also that by contracting $i$ with $j$ in (106) we obtain for higher dimensions the same relation which is also valid for $4 \mathrm{D}$

$$
D S=A^{2}-S^{2} \text {. }
$$

\section{Discussion}

In this paper, we present a higher dimensional frame formalism. The complete set of frame components of the Bianchi identities, which are in this context usually called the Bianchi equations, is given in Appendix B. For algebraically general spacetimes these equations are quite complicated. However, for algebraically special cases they are much simpler (e.g., see Section 3 for the type $\mathrm{N}$ case). In $4 \mathrm{D}$ it is possible to use the Bianchi and Ricci equations to construct many algebraically special solutions of Einstein's field equations. The hope is that it is possible to do a similar thing in higher dimensions, at least for the simplest algebraically special spacetimes. The vast majority of today's known higher dimensional exact solutions are simple generalizations of 4D solutions. The present approach may lead to new, genuinely higher dimensional exact solutions. Type $\mathrm{N}$ and D (see [1, 3] for the definition of type D in higher dimensions) solutions may be of particular physical interest.

In particular, we study the consequences of the algebraic Bianchi equations for type $\mathrm{N}$ and III spacetimes. It turns out that the principal null direction $\ell$ in these spacetimes is geodesic. For vacuum type $\mathrm{N}$ spacetimes with non-vanishing expansion or twist we also prove that the corresponding components of the Weyl tensor and expansion and twist matrices, in an appropriately chosen frame, can be expressed as

$\Psi_{i j}=\sqrt{\frac{p}{2}}\left(v_{i} v_{j}-w_{i} w_{j}\right), \quad S_{i j}=S\left(v_{i} v_{j}+w_{i} w_{j}\right), \quad A_{k l}=A\left(w_{k} v_{l}-v_{k} w_{l}\right)$.

Note that we do not obtain any constraints for $\Psi_{i j}$ in non-twisting and non-expanding type $\mathrm{N}$ spacetimes. We also establish similar results for vacuum type III spacetimes.

The Weyl tensor is said to be reducible if we can decompose it into two parts

$$
C_{b c d}^{a}=C_{\tilde{b} \tilde{c} \tilde{d}}^{\tilde{a}}+C_{\hat{b} \hat{c} \hat{d}}^{\hat{a}}
$$

where indices $a, b, \ldots$ have values from 0 to $n-1$, whereas indices $\tilde{a}, \tilde{b}, \ldots$ have values from 0 to $N-1$, and indices $\hat{a}, \hat{b}, \ldots$ from $\mathrm{N}$ to $n-1[\underline{3}$. In this sense the Weyl tensor in vacuum type $\mathrm{N}$ spacetimes with non-vanishing expansion or twist is reducible, with a nontrivial four-dimensional part and a vanishing $(n-4)$-dimensional part.

In 4D the well-known Goldberg-Sachs theorem [4] states that 'a vacuum metric is algebraically special if and only if it contains a shearfree geodesic null congruence'. This theorem (and also its non-vacuum generalizations - see [5]) is very useful for constructing algebraically special exact solutions. At present it is unclear if and to what extent this theorem may be generalized for higher dimensions (see Sec. 5 for details).

Recently, all 4D spacetimes with vanishing curvature invariants (i.e., vanishing invariants constructed from the Riemann tensor and its covariant derivatives of an arbitrary order - VSI spacetimes) were determined in [6]. In [7, the generalization to higher dimensions was discussed. All these spacetimes are of type III, N or O. The results presented in this paper will enable us to explicitly express curvature invariants involving derivatives of the Riemann tensor in higher dimensions. For example, for type $\mathrm{N}$ vacuum spacetimes we obtain, thanks to Lemma 1 ,

$$
I=C^{a b c d ; p q} C_{a m c n ; p q} C^{t m u n ; r s} C_{t b u d ; r s}=3^{2} 2^{10} p^{2}\left(S^{2}+A^{2}\right)^{4} .
$$


Consequently, we will be able to prove that higher dimensional VSI spacetimes are expansion and twist free, thereby proving the assertion made in [7] (see [8]).

\section{Acknowledgments}

We are grateful to the referee for constructive criticism. AP and VP would like to thank Dalhousie University for its hospitality while part of this work was carried out. RM and AC were partially supported by a research grant from NSERC. VP was supported by grant GACR-202/03/P017 and AP by grant KJB1019403. AP and VP also thank to NČLF for support.

\section{Appendix A. Components of equations (54) and (55)}

Equation (54) gives

$$
\begin{aligned}
& \mathcal{C}_{\alpha[\beta} b_{\gamma]}+\sum_{F} \mathcal{B}_{F[\beta \mid} \mathcal{P}_{F \alpha \mid \gamma]}+4 \mathcal{C}_{\phi[\beta \mid} \mathcal{S}_{\phi \alpha \mid \gamma]}=0, \\
& 2 \mathcal{C}_{\alpha \beta} a_{C}+\mathcal{B}_{C \alpha} b_{\beta}-2 \lambda_{C} \mathcal{P}_{C \alpha \beta}+\sum_{F} 4 \mathcal{A}_{C F} \mathcal{P}_{F \alpha \beta}+2 \sum_{F} \mathcal{B}_{F \beta} \mathcal{O}_{F \alpha C} \\
& +8 \mathcal{C}_{\phi \beta} \mathcal{R}_{\phi \alpha C}+4 \mathcal{B}_{C \phi} \mathcal{S}_{\phi \alpha \beta}=0, \\
& \mathcal{B}_{A[\beta} b_{\gamma]}+4 \sum_{F} \mathcal{B}_{F[\beta \mid} \mathcal{N}_{F A \mid \gamma]}-4 \mathcal{C}_{\phi[\beta \mid} \mathcal{P}_{A \phi \mid \gamma]}=0, \\
& \lambda_{B} \mathcal{O}_{B \alpha C}-\lambda_{C} \mathcal{O}_{C \alpha B}+\mathcal{B}_{[C \mid \alpha} a_{\mid B]}+4 \sum_{F} \mathcal{A}_{F[B \mid} \mathcal{O}_{F \alpha \mid C]}+4 \mathcal{B}_{[B \mid \phi} \mathcal{R}_{\alpha \phi \mid C]}=0, \\
& \lambda_{A} \delta_{A B} b_{\gamma}-\mathcal{B}_{A \gamma} a_{B}+2 \mathcal{A}_{A B} b_{\gamma}+4 \lambda_{B} \mathcal{N}_{B A \gamma}+8 \sum_{F} \mathcal{A}_{F B} \mathcal{N}_{F A \gamma} \\
& +2 \mathcal{B}_{B \phi} \mathcal{P}_{A \phi \gamma}-4 \sum_{F} \mathcal{B}_{F \gamma} \mathcal{M}_{F A B}+4 \mathcal{C}_{\phi \gamma} \mathcal{O}_{A \phi B}=0, \\
& \lambda_{A} \delta_{A[B} a_{C]}+2 \mathcal{A}_{A[B} a_{C]}+2 \lambda_{B} \mathcal{M}_{B A C}-2 \lambda_{C} \mathcal{M}_{C A B} \\
& +8 \sum_{F} \mathcal{A}_{F[B \mid} \mathcal{M}_{F A \mid C]}+2 \mathcal{B}_{[B \mid \phi} \mathcal{O}_{A \phi \mid C]}=0 .
\end{aligned}
$$

Equation (55) leads to

$$
\begin{aligned}
& 2 \mathcal{C}_{\{\alpha \beta \mid} \mathcal{S}_{\gamma \delta \mid \varepsilon\}}+\mathcal{C}_{\delta\{\alpha} \mathcal{S}_{\beta \varepsilon\} \gamma}-\mathcal{C}_{\gamma\{\alpha} \mathcal{S}_{\beta \varepsilon\} \delta}=0 \\
& 2 \mathcal{C}_{\beta \varepsilon} \mathcal{R}_{\gamma \delta A}+\mathcal{C}_{\delta[\varepsilon \mid} \mathcal{P}_{A \mid \beta] \gamma}+\mathcal{C}_{\gamma[\beta \mid} \mathcal{P}_{A \mid \varepsilon] \delta}+2 \mathcal{B}_{A[\beta} \mathcal{S}_{\gamma \delta \mid \varepsilon]}+\mathcal{B}_{A[\gamma} \mathcal{S}_{\beta \varepsilon \mid \delta]}=0, \\
& 2 \mathcal{C}_{\{\alpha \beta \mid} \mathcal{P}_{C \delta \mid \varepsilon\}}+2 \mathcal{C}_{\delta\{\alpha} \mathcal{R}_{\beta \varepsilon\} C}-\mathcal{B}_{C\{\alpha} \mathcal{S}_{\beta \varepsilon\} \delta}=0 \\
&-4 \mathcal{B}_{[A \mid \varepsilon} \mathcal{R}_{\gamma \delta \mid B]}+\mathcal{B}_{[B \mid \delta} \mathcal{P}_{\mid A] \varepsilon \gamma}-\mathcal{B}_{[B \mid \gamma} \mathcal{P}_{\mid A] \varepsilon \delta}+4 \mathcal{C}_{[\delta \mid \varepsilon} \mathcal{N}_{A B \mid \gamma]}+4 \mathcal{A}_{A B} \mathcal{S}_{\gamma \delta \varepsilon}=0, \\
& 2 \mathcal{B}_{A[\beta \mid} \mathcal{P}_{C \delta \mid \varepsilon]}+\mathcal{B}_{C[\beta \mid} \mathcal{P}_{A \mid \varepsilon] \delta}+2 \mathcal{C}_{\beta \varepsilon} \mathcal{O}_{C \delta A}-2 \mathcal{C}_{\delta[\beta \mid} \mathcal{O}_{A \mid \varepsilon] C}-\mathcal{B}_{A \delta} \mathcal{R}_{\beta \varepsilon C} \\
& \quad-\left(\lambda_{A} \delta_{A C}+2 \mathcal{A}_{C A}\right) \mathcal{S}_{\beta \varepsilon \delta}=0 \\
& 4 \mathcal{C}_{\{\alpha \beta \mid} \mathcal{N}_{C D \mid \varepsilon\}}+\mathcal{B}_{D\{\alpha} \mathcal{R}_{\beta \varepsilon\} C}-\mathcal{B}_{C\{\alpha} \mathcal{R}_{\beta \varepsilon\} D}=0 \\
& 4 \mathcal{C}_{\alpha \beta} \mathcal{M}_{C D E}+4 \mathcal{B}_{E[\alpha \mid} \mathcal{N}_{C D \mid \beta]}+\mathcal{B}_{D[\beta \mid} \mathcal{O}_{E \mid \alpha] C}-\mathcal{B}_{C[\beta \mid} \mathcal{O}_{E \mid \alpha] D} \\
& \quad+2 \lambda_{E} \delta_{E[D} \mathcal{R}_{\alpha \beta \mid C]}+4 \mathcal{A}_{[D \mid E} \mathcal{R}_{\alpha \beta \mid C]}=0 \\
& 2 \mathcal{C}_{\alpha \gamma} \mathcal{M}_{B E D}+\mathcal{B}_{D \alpha} \mathcal{N}_{B E \gamma}+2 \mathcal{B}_{[B \mid \alpha} \mathcal{O}_{D \gamma \mid E]}+\mathcal{B}_{[B \mid \gamma} \mathcal{O}_{E \alpha \mid D]} \\
&-2 \mathcal{A}_{B E} \mathcal{P}_{D \gamma \alpha}+2 \mathcal{A}_{D[B} \mathcal{P}_{E] \alpha \gamma}+\lambda_{D} \delta_{D[B} \mathcal{P}_{E] \alpha \gamma}=0 \\
& \mathcal{B}_{[A \mid \gamma} \mathcal{N}_{B] E \delta}+\mathcal{B}_{[B \mid \delta} \mathcal{N}_{A] E \gamma}+\mathcal{B}_{E[\gamma} \mathcal{N}_{A B \mid \delta]}+2 \mathcal{A}_{\{A B \mid} \mathcal{R}_{\gamma \delta \mid E\}}=0,
\end{aligned}
$$




$$
\begin{aligned}
4 \mathcal{B}_{[E \mid \alpha} \mathcal{M}_{C D \mid B]}+ & 2 \mathcal{B}_{[D \mid \alpha} \mathcal{M}_{B E \mid C]}+4 \mathcal{A}_{B E} \mathcal{N}_{C D \alpha}+2 \mathcal{A}_{D[B} \mathcal{O}_{E] \alpha C}+2 \mathcal{A}_{C[E} \mathcal{O}_{B] \alpha D} \\
+\lambda_{B} \delta_{B[D \mid} \mathcal{O}_{E \alpha \mid C]} & +\lambda_{E} \delta_{E[C \mid} \mathcal{O}_{B \alpha \mid D]}=0 \\
\mathcal{B}_{\{A \mid \gamma} \mathcal{M}_{B E\} D}- & 2 \mathcal{A}_{\{A B \mid} \mathcal{O}_{D \gamma \mid E\}}+2 \mathcal{A}_{D\{A} \mathcal{N}_{B E\} \gamma}+\lambda_{D} \delta_{D\{A} \mathcal{N}_{B E\} \gamma}=0 \\
4 \mathcal{A}_{\{A B \mid} \mathcal{M}_{C D \mid E\}}+ & 2 \mathcal{A}_{D\{A} \mathcal{M}_{B E\} C}-2 \mathcal{A}_{C\{A} \mathcal{M}_{B E\} D} \\
& +\lambda_{D} \delta_{D\{A} \mathcal{M}_{B E\} C}-\lambda_{C} \delta_{C\{A} \mathcal{M}_{B E\} D}=0
\end{aligned}
$$

\section{Appendix B. Bianchi equations}

$$
\begin{aligned}
& D R_{101 i}+\triangle R_{010 i}-\delta_{i} R_{0101}=2 R_{010[i} L_{1] 1}-R_{101 i} L_{10} \\
& -R_{01 i s} L_{s 1}-2 R_{101 s} L_{s i}+2 R_{[1 \mid i 1 s} L_{s \mid 0]}-R_{0101} N_{i 0}-2 R_{010 s} N_{s i} \\
& +R_{01 i s} N_{s 0}+2 R_{0 s[0 \mid i} N_{s \mid 1]}-R_{010 s} \stackrel{s}{M}{ }_{i 1}-R_{101 s} \stackrel{s}{M}{ }_{i 0}, \\
& -\triangle R_{01 i j}+2 \delta_{[j \mid} R_{101 \mid i]}=2 R_{101[j \mid} L_{1 \mid i]}+2 R_{101[i \mid} L_{\mid j] 1}+2 R_{1[i \mid 1 s} L_{s \mid j]}+R_{1 s i j} L_{s 1} \\
& +2 R_{0101} N_{[j i]}+2 R_{010[j} N_{i] 1}+2 R_{01[i \mid s} N_{s \mid j]}+2 R_{0 s 1[j \mid} N_{s \mid i]}-R_{0 s i j} N_{s 1} \\
& \left.+2 R_{01[i \mid s} \stackrel{s}{M} \mid j\right] 1+2 R_{101 s} \stackrel{s}{M}[j i], \\
& -D R_{01 i j}+2 \delta_{[i \mid} R_{010 \mid j]}=2 R_{0101} L_{[i j]}+2 R_{010[j \mid} L_{1 \mid i]}+2 R_{01[i \mid s} L_{s \mid j]} \\
& +2 R_{0[i \mid 1 s} L_{s \mid j]}+2 R_{101[i} L_{j] 0}+R_{1 s i j} L_{s 0}+2 R_{010[j} N_{i] 0}+2 R_{0[j \mid 0 s} N_{s \mid i]} \\
& \left.-R_{0 s i j} N_{s 0}+2 R_{01[i \mid s} \stackrel{s}{M} \mid j\right] 0+2 R_{010 s} \stackrel{s}{M}_{[i j]} \text {, } \\
& D R_{1 i 1 j}-\triangle R_{0 j 1 i}-\delta_{j} R_{101 i}=2 R_{101 i} L_{[1 j]}-2 R_{1 i 1 j} L_{10}+2 R_{1 i[j \mid s} L_{s \mid 1]} \\
& +2 R_{010[1 \mid} N_{i \mid j]}-2 R_{101(i \mid} N_{\mid j) 0}+2 R_{0[j \mid i s} N_{s \mid 1]}+R_{0 s 1 i} N_{s j}-2 R_{1(i j) s} N_{s 0}
\end{aligned}
$$

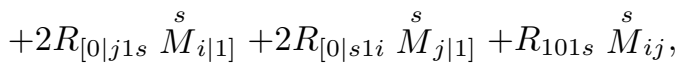

$$
\begin{aligned}
& D R_{0 i 1 j}-\triangle R_{0 i 0 j}+\delta_{j} R_{010 i}=2 R_{010[j \mid} L_{i \mid 1]}+2 R_{010 i} L_{(1 j)}-2 R_{0 i 0 j} L_{11} \\
& +R_{101 j} L_{i 0}+2 R_{0 i[j \mid s} L_{s \mid 1]}+2 R_{0[j \mid i s} L_{s \mid 1]}-R_{1 j i s} L_{s 0}+R_{010 i} N_{j 0}+2 R_{0 i[0 \mid s} N_{s \mid j]} \\
& -R_{010 s} \stackrel{s}{M} i j+2 R_{0 s[0 \mid j} \stackrel{s}{M} \text { i|1] }+2 R_{0 i[0 \mid s} \stackrel{s}{M} \text { j|1] } \\
& -D R_{1 k i j}+\triangle R_{0 k i j}-\delta_{k} R_{01 i j}=-2 R_{0[1 \mid i j} L_{\mid k] 1}+2 R_{0 k 1[i} L_{j] 1}+2 R_{101[i} L_{j] k} \\
& -2 R_{1[i \mid 1 k} L_{\mid j] 0}+R_{1 k i j} L_{10}+2 R_{[1 \mid s i j} L_{s \mid k]}-2 R_{010[i} N_{j] k}-R_{01 i j} N_{k 0} \\
& +2 R_{0[i \mid 0 k} N_{\mid j] 1}+2 R_{0[j \mid 1 k} N_{\mid i] 0}+2 R_{[k \mid s i j} N_{s \mid 0]} \\
& +2 R_{01[i \mid s} \stackrel{s}{M}[j] k+2 R_{[0 \mid k j s} \stackrel{s}{M}{ }_{i \mid 1]}+2 R_{[1 \mid k i s} \stackrel{s}{M}{ }_{j \mid 0]}+2 R_{[1 \mid s i j} \stackrel{s}{M} k \text { k|0] }, \\
& \triangle R_{0 i j k}+2 \delta_{[k \mid} R_{0 i 1 \mid j]}=2 R_{0 i 1[j \mid} L_{\mid k] 1}+2 R_{0[i \mid j k} L_{\mid 1] 1}+2 R_{101[j \mid} L_{i \mid k]}+2 R_{1[k \mid i s} L_{s \mid j]} \\
& -R_{i s j k} L_{s 1}+2 R_{010 i} N_{[j k]}+2 R_{0 i 0[j} N_{k] 1}+2 R_{0 i[k \mid s} N_{s \mid j]} \\
& \left.+2 R_{0 i 1 s} \stackrel{s}{M}_{[k j]}+2 R_{0 s 1[k \mid} \stackrel{s}{M}_{i \mid j]}+2 R_{0 i[k \mid s} \stackrel{s}{M} \mid j\right] 1-R_{0 s j k} \stackrel{s}{M}{ }_{i 1}, \\
& D R_{0 i j k}+2 \delta_{[k \mid} R_{0 i 0 \mid j]}=-2 R_{010 i} L_{[j k]}+2 R_{010[k \mid} L_{i \mid j]}+4 R_{0 i 0[j \mid} L_{1 \mid k]}+2 R_{0 i 1[j} L_{k] 0} \\
& +2 R_{0[i \mid j k} L_{\mid 1] 0}+2 R_{0 i[k \mid s} L_{s \mid j]}+2 R_{0[k \mid i s} L_{s \mid j]}-R_{i s j k} L_{s 0}+2 R_{0 i 0[j \mid} N_{\mid k] 0} \\
& \left.+2 R_{0 i 0 s} \stackrel{s}{M}[k j]+2 R_{0[k \mid 0 s} \stackrel{s}{M}{ }_{i \mid j]}+2 R_{0 i[k \mid s} \stackrel{s}{M} \mid j\right] 0-R_{0 s j k} \stackrel{s}{M}{ }_{i 0},
\end{aligned}
$$




$$
\begin{aligned}
& D R_{1 i j k}+2 \delta_{[k \mid} R_{0 \mid j] 1 i}=2 R_{101 i} L_{[j k]}+2 R_{1 i 1[j} L_{k] 0}-R_{1 i j k} L_{10}+2 R_{1 i[k \mid s} L_{s \mid j]} \\
& +R_{010 j} N_{i k}+2 R_{01[j \mid k} N_{i \mid 0]}-2 R_{0[k \mid 1 i} N_{\mid j] 0}+2 R_{0[k \mid i s} N_{s \mid j]}-R_{i s j k} N_{s 0}
\end{aligned}
$$

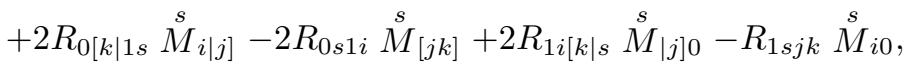

$$
\begin{aligned}
& \triangle R_{1 i j k}+2 \delta_{[k \mid} R_{1 i 1 \mid j]}=2 R_{1 i 1[j} L_{k] 1}+4 R_{1 i 1[k \mid} L_{1 \mid j]}-R_{1 i j k} L_{11} \\
& +R_{01 j k} N_{i 1}+2 R_{0[j \mid 1 i} N_{\mid k] 1}+2 R_{101[k \mid} N_{i \mid j]}+2 R_{101 i} N_{[k j]}+2 R_{1 i[k \mid s} N_{s \mid j]}+2 R_{1[k \mid i s} N_{s \mid j]} \\
& \left.-R_{i s j k} N_{s 1}-2 R_{1 i 1 s} \stackrel{s}{M}[j k]+2 R_{1[k \mid 1 s} \stackrel{s}{M}{ }_{i \mid j]}+2 R_{1 i[k \mid s} \stackrel{s}{M} \mid j\right] 1-R_{1 s j k} \stackrel{s}{M} \text { i1 } \text {, } \\
& -\delta_{\{i \mid} R_{01 \mid j k\}}=R_{101\{i} L_{j k\}}-R_{101\{i} L_{k j\}}+R_{1 s\{i j \mid} L_{s \mid k\}}+R_{010\{i} N_{k j\}} \\
& \left.-R_{010\{i} N_{j k\}}-R_{0 s i j} N_{s k}+2 R_{0 s[i \mid k} N_{s \mid j]}+R_{01\{i \mid s} \stackrel{s}{M} \mid j k\right\}-R_{01\{i \mid s} \stackrel{s}{M \mid k j\}}, \\
& -D R_{i j k m}+2 \delta_{[k \mid} R_{0 \mid m] i j}=2 R_{01 i j} L_{[k m]}+2 R_{0 k[1 \mid j} L_{\mid i] m}+2 R_{0[m \mid 1 i} L_{j \mid k]} \\
& +2 R_{0 m[i \mid j} L_{\mid 1] k}+2 R_{1[j \mid k m} L_{\mid i] 0}+2 R_{1[m \mid i j} L_{\mid k] 0}+2 R_{i j[k \mid s} L_{s \mid m]} \\
& +2 R_{0 i 0[m \mid} N_{j \mid k]}+2 R_{0 j 0[k \mid} N_{i \mid m]}+2 R_{0[j \mid k m} N_{\mid i] 0}+2 R_{0[m \mid i j} N_{\mid k] 0}+2 R_{0[k \mid i s} \stackrel{s}{M}{ }_{j \mid m]} \\
& +2 R_{0[m \mid j s} \stackrel{s}{M}{ }_{i \mid k]}+2 R_{0 s i j} \stackrel{s}{M}[k m]+2 R_{[i \mid s k m} \stackrel{s}{M}{ }_{\mid j] 0}+2 R_{i j[k \mid s} \stackrel{s}{M}{ }_{\mid m] 0}, \\
& -\triangle R_{i j k m}+2 \delta_{[k \mid} R_{1 \mid m] i j}=2 R_{1 i[1 \mid m} L_{j \mid k]}+2 R_{1[j \mid 1 k} L_{\mid i] m}+2 R_{1 j[k \mid m} L_{i \mid 1]} \\
& +2 R_{1 k i j} L_{[1 m]}+2 R_{1 m i j} L_{[k 1]}-2 R_{01 i j} N_{[k m]}+2 R_{0[i \mid 1 m} N_{\mid j] k}+2 R_{0[j \mid 1 k} N_{\mid i] m} \\
& +2 R_{0[j \mid k m} N_{\mid i] 1}+2 R_{0[m \mid i j} N_{\mid k] 1}+2 R_{i j[k \mid s} N_{s \mid m]}+2 R_{1 k[i \mid s} \stackrel{s}{M \mid j] m} \\
& +2 R_{1 m[j \mid s} \stackrel{s}{M}_{\mid i] k}+2 R_{1 s i j} \stackrel{s}{M}_{[k m]}+2 R_{i j[k \mid s} \stackrel{s}{M} \mid \begin{array}{l}
\mid m] 1 \\
D
\end{array} R_{[i \mid s k m} \stackrel{s}{M}_{\mid j] 1}, \\
& \delta_{\{j \mid} R_{1 i \mid m k\}}=R_{1 i 1\{j} L_{m k\}}-R_{1 i 1\{j} L_{k m\}}+R_{1 i\{j k \mid} L_{1 \mid m\}}+R_{01\{j m \mid} N_{i \mid k\}}-R_{0\{j \mid 1 i} N_{\mid k m\}}
\end{aligned}
$$

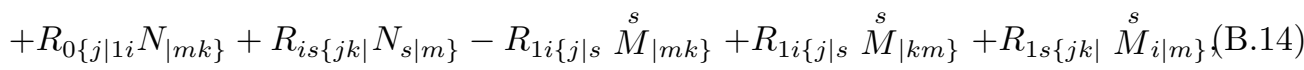

$$
\begin{aligned}
& \delta_{\{j \mid} R_{0 i \mid m k\}}=R_{01\{j k \mid} L_{i \mid m\}}-R_{0 i 1\{j} L_{k m\}}+R_{0 i 1\{j} L_{m k\}}+R_{0 i\{j m \mid} L_{1 \mid k\}}+R_{i s\{j k \mid} L_{s \mid m\}} \\
& \left.+R_{0 i 0\{j} N_{m k\}}-R_{0 i 0\{j} N_{k m\}}+R_{0 i\{j \mid s} \stackrel{s}{M \mid k m\}}-R_{0 i\{j \mid s} \stackrel{s}{M} \mid m k\right\}+R_{0 s\{j k \mid} \stackrel{s}{M}{ }_{i \mid m\}}, \\
& \delta_{\{k \mid} R_{i j \mid n m\}}=R_{1 j\{k m \mid} L_{i \mid n\}}-R_{1 i\{k m \mid} L_{j \mid n\}}-R_{1\{k \mid i j} L_{\mid m n\}}+R_{1\{k \mid i j} L_{\mid n m\}} \\
& +R_{0 j\{k m \mid} N_{i \mid n\}}-R_{0 i\{k m \mid} N_{j \mid n\}}+R_{0\{k \mid i j} N_{\mid n m\}}-R_{0\{k \mid i j} N_{\mid m n\}}
\end{aligned}
$$

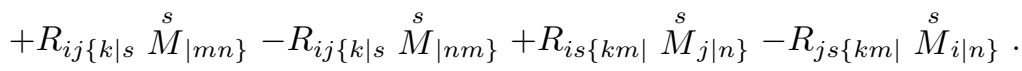

\section{Appendix C. Proof for vacuum type III spacetimes}

Appendix C.1. Non-twisting case in an arbitrary dimension

In this case, $\boldsymbol{A}=0$ and thus $\boldsymbol{L}=\boldsymbol{S}$ is symmetric (we assume $\boldsymbol{S} \neq 0$ since in the non-twisting and non-expanding case both sides of equations (54) and (55) vanish).

From (60) we obtain

$$
S_{i j} \Psi_{j}=\frac{L}{2} \Psi_{i} .
$$


Note that providing $\Psi_{i} \neq 0$ from (C.1) and from (67) we obtain $L^{2}=2 \ell$ and thus the case $L=0$ leads to $\boldsymbol{S}=0$.

Equation (59) leads to

$$
L \Psi_{i j k}=2 \Psi_{i j s} S_{s k},
$$

which using the decomposition (69) implies

$$
L \mathcal{N}_{A B \gamma}=0, \quad L \mathcal{P}_{A \beta \gamma}=0, \quad L \mathcal{S}_{\alpha \beta \gamma}=0
$$

$\left(L-2 \lambda_{C}\right) \mathcal{M}_{A B C}=0, \quad\left(L-2 \lambda_{C}\right) \mathcal{O}_{A \beta C}=0, \quad\left(L-2 \lambda_{C}\right) \mathcal{R}_{\alpha \beta C}=0$.

First study the case with $L=0$. From equations (C.3) we get $\mathcal{M}_{A B C}=0$, $\mathcal{O}_{A \beta C}=0, \mathcal{R}_{\alpha \beta C}=0$ and thus from (76) and (81) $\mathcal{P}_{A \beta \gamma}=0, \mathcal{N}_{A B \gamma}=0$ and further from (A.11) $\mathcal{S}_{\alpha \beta \gamma}=0$ which imply $\Psi_{i j k}=0$, i.e., a type $\mathrm{N}$ or O spacetime and thus there are no non-trivial solutions of (54) and (55) of type III with $L=0$.

Now we may proceed to the case $L \neq 0$. Then (C.3) and (80) imply

$$
\mathcal{N}_{A B \gamma}=0, \mathcal{P}_{A \beta C}=0, \mathcal{S}_{\alpha \beta \gamma}=0, \mathcal{R}_{\alpha \beta C}=0 .
$$

From (C.1) we get

$$
\lambda_{A} a_{A}=\frac{L}{2} a_{A}, \quad \text { and } \quad b_{\alpha}=0,
$$

which leads to

$$
a_{A}=0 \text { or } \lambda_{A}=\frac{L}{2} \text {. }
$$

(i) If all $a_{A} \neq 0$ then all $\lambda_{A}=L / 2\left(\right.$ C.6) and thus $A_{\max }=2, \lambda_{1}=\lambda_{2}=L / 2$. The corresponding solution is given by (C.20).

(ii) If at least one $a_{A} \neq 0$ then corresponding $\lambda_{A}=L / 2$ and from (79) with $B=C \neq A$ and (A.6) with $A=C \neq B$ and after interchanging $A$ with $B$ we get

$$
\begin{aligned}
a_{A} & =\left.\frac{2 L-4 \lambda_{B}+4 \lambda_{A}}{\lambda_{B}} \mathcal{M}_{A B B}\right|_{B \neq A}, \\
a_{A} & =\left.4 \frac{\lambda_{A}}{\lambda_{B}} \mathcal{M}_{A B B}\right|_{B \neq A} .
\end{aligned}
$$

Comparing the right hand sides we obtain $2 L-4 \lambda_{B}+4 \lambda_{A}=4 \lambda_{A}$ and thus $\lambda_{B}=L / 2$ for all $B \neq A$. However, the remaining $\lambda_{A}=L / 2$ and in this case again $A_{\max }=2, \lambda_{1}=\lambda_{2}=L / 2$.

(iii) If all $a_{A}=0$ then (C.8) implies

$$
\mathcal{M}_{A B B}=0
$$

for all $A, B$. We treat separately the case with all $\mathcal{M}_{A B C}=0$ and the case with some $\mathcal{M}_{A B C} \neq 0$.

(A)

If all $\mathcal{M}_{A B C}=0$ than there exists at least one non-vanishing $\mathcal{O}_{A \beta C}$ in order to have a type III Weyl tensor.

For $A_{\max } \geq 3$, from equation (A.16) for $B=C, C \neq E, C \neq D, D \neq E$ we get $\lambda_{C} \mathcal{O}_{E \alpha D}=0$ which implies that all $\mathcal{O}_{E \alpha D}=\left.0\right|_{E \neq D}$. Equation (77) with $C=A$, i.e., $\left(L-2 \lambda_{A}\right) \mathcal{O}_{A \beta A}=0$, implies

$$
\mathcal{O}_{A \beta A}=0 \text { or } \lambda_{A}=\frac{L}{2} \text {. }
$$


From equation (A.16) (for $B=C, D=E, C \neq E$ )

$$
\lambda_{C} \mathcal{O}_{D \alpha D}=-\left.\lambda_{D} \mathcal{O}_{C \alpha C}\right|_{D \neq C} .
$$

If there exists $\lambda_{C} \neq L / 2$ then from (C.10) $\mathcal{O}_{C \alpha C}=0$ and thus from (C.11) all $\mathcal{O}_{D \alpha D}=0$ and $\Psi_{i j k}=0$, the spacetime is of the type $\mathrm{N}$ or $\mathrm{O}$. The other possibility that all $\lambda_{A}=L / 2$ is not compatible with $A_{\max } \geq 3$ and so $A_{\max } \leq 2$.

For $A_{\max }=2$, (71) with (C.11) imply

$$
\lambda_{2}=\lambda_{1}=\frac{L}{2} \text { and } \mathcal{O}_{2 \beta 2}=-\mathcal{O}_{1 \beta 1}
$$

or $\mathcal{O}_{2 \beta 2}=\mathcal{O}_{1 \beta 1}=0$. In the second case, equation (81) implies

$$
\mathcal{O}_{2 \beta 1}=\mathcal{O}_{1 \beta 2}
$$

and from (78) (or (A.4) ) we get $\lambda_{2}=\lambda_{1}=L / 2$.

For $A_{\max }=1$, from (C.10) we immediately get $\mathcal{O}_{1 \beta 1}=0$, type $\mathrm{N}$ or $\mathrm{O}$ spacetimes. To summarize, the case $\mathcal{M}_{A B C}=0(\forall A, B, C)$ always leads to $A_{\max }=2$, $\lambda_{1}=\lambda_{2}=L / 2$ or to type $\mathrm{N}$ or $\mathrm{O}$ spacetimes.

\section{(B)}

Let us now assume, for $A_{\max } \geq 4$, that there exists $\mathcal{M}_{A B C} \neq\left. 0\right|_{B \neq C}$. Then (A.18) for $C \neq D, A \neq D, A \neq C, B=D, C \neq E$ and $D \neq E$ implies $\lambda_{D} \mathcal{M}_{A E C}=0$ and thus all $\mathcal{M}_{A B C}=\left.0\right|_{B \neq C}$ which is in contradiction with our assumptions and consequently $A_{\max } \leq 3$.

Equation (79) with $C \neq A$ and $B \neq C$ and (A.6) gives

$$
\begin{aligned}
\left(L-2 \lambda_{C}+2 \lambda_{A}\right) \mathcal{M}_{C A B} & =\left.\left(L-2 \lambda_{C}+2 \lambda_{B}\right) \mathcal{M}_{C B A}\right|_{C \neq A, C \neq B}, \\
\lambda_{A} \mathcal{M}_{C A B} & =\lambda_{B} \mathcal{M}_{C B A} .
\end{aligned}
$$

These two equations leads to

$$
\left(L-2 \lambda_{C}\right) \mathcal{M}_{C A B}=\left(L-2 \lambda_{C}\right) \mathcal{M}_{C B A}
$$

and so either

$$
\lambda_{C}=\frac{L}{2} \wedge \lambda_{A} \mathcal{M}_{C A B}=\lambda_{B} \mathcal{M}_{C B A}
$$

or

$\lambda_{C} \neq \frac{L}{2} \wedge \mathcal{M}_{C A B}=\mathcal{M}_{C B A} \wedge \lambda_{A} \mathcal{M}_{C A B}=\lambda_{B} \mathcal{M}_{C B A}$

i.e.,

$\mathcal{M}_{C A B}=\mathcal{M}_{C B A}=0$ or $\mathcal{M}_{C A B}=\mathcal{M}_{C B A} \neq 0 \wedge \lambda_{A}=\lambda_{B}$.

Let us assume that $A_{\max }=3$. Let $\lambda_{1} \neq L / 2$. Then the case (a) $\mathcal{M}_{123}=\mathcal{M}_{132}=0$ (C.18) implies $\mathcal{M}_{231}=0$ (82) which is the case $\mathcal{M}_{A B C}=0$ studied above (A). The condition (b) $\mathcal{M}_{123}=\mathcal{M}_{132} \neq 0$ (C.18) also implies $\mathcal{M}_{231}=0$ (82) and for $\lambda_{2} \neq L / 2$ we get $\mathcal{M}_{213}=\mathcal{M}_{231}=0$ (C.18) and thus also $\mathcal{M}_{123}=0$ and this is again the case $\mathcal{M}_{A B C}=0$. However, if $\lambda_{2}=L / 2$ then $\lambda_{1} \mathcal{M}_{213}=\lambda_{3} \mathcal{M}_{231}=0$ (C.17) and since $\mathcal{M}_{231}=0$ we again obtain the case $\mathcal{M}_{A B C}=0$.

If $\lambda_{1}=L / 2$ then $\lambda_{2}=L / 2$ immediately implies $\lambda_{3}=0$ and $\lambda_{2} \neq L / 2$ corresponds to the case analyzed in the previous paragraph.

$A_{\max }<3$ again leads to all $\mathcal{M}_{A B C}=0$ (C.9). 
To summarize, the general solution of (54) and (55) in the non-twisting case may be written, substituting (C.4), (C.5), (C.8), (C.12) and (C.13) into (69), in the form

$$
\begin{aligned}
\Psi_{i} & =4 \mathcal{M}_{122} v_{i}^{1}+4 \mathcal{M}_{211} v_{i}^{2}, \\
S_{i j} & =\frac{L}{2}\left(v_{i}^{1} v_{j}^{1}+v_{i}^{2} v_{j}^{2}\right), \\
\Psi_{i j k} & =2 \mathcal{M}_{122}\left(v_{i}^{1} v_{j}^{2}-v_{j}^{1} v_{i}^{2}\right) v_{k}^{2}+2 \mathcal{M}_{211}\left(v_{i}^{2} v_{j}^{1}-v_{j}^{2} v_{i}^{1}\right) v_{k}^{1} \\
& +\mathcal{O}_{1 \alpha 1}\left(v_{i}^{1} u_{j}^{\alpha}-v_{j}^{1} u_{i}^{\alpha}\right) v_{k}^{1}-\mathcal{O}_{1 \alpha 1}\left(v_{i}^{2} u_{j}^{\alpha}-v_{j}^{2} u_{i}^{\alpha}\right) v_{k}^{2} \\
& +\mathcal{O}_{1 \alpha 2}\left(v_{i}^{1} u_{j}^{\alpha}-v_{j}^{1} u_{i}^{\alpha}\right) v_{k}^{2}+\mathcal{O}_{1 \alpha 2}\left(v_{i}^{2} u_{j}^{\alpha}-v_{j}^{2} u_{i}^{\alpha}\right) v_{k}^{1} .
\end{aligned}
$$

\section{Appendix C.2. Twisting type III spacetimes in five dimensions}

In Section 4 we studied possible non-zero eigenvalues $\lambda_{A}, A=1, \ldots, A_{\max }$, of $\boldsymbol{S}$ which are compatible with equations (54) and (55), providing that for every pair $A$, $C(A \neq C)$ there exists $\beta$ for which $\mathcal{O}_{A \beta C} \neq 0$. It turned out that then it follows from (54) and (55) that $A_{\max }=2$ and $\lambda_{1}=\lambda_{2}=L / 2$. Here we study all other possible cases in 5D and show that the only case with non-vanishing eigenvalues of $\boldsymbol{S}$ is $A_{\max }=2$ and $\lambda_{1}=\lambda_{2}=L / 2$. Corresponding form of $\Psi_{i j k}, \Psi_{i}, \boldsymbol{S}$ and $\boldsymbol{A}$ is given in Section 4 .

In $5 \mathrm{D}, A_{\max }$ can have values $0,1,2$ or 3 . We will treat these cases separately (the cases $A_{\max }=0$ and $A_{\max }=1$ can be easily solved in an arbitrary dimension).

(i) The case $A_{\max }=0$ (globally) corresponds to $\boldsymbol{S}=0$ and one of the Ricci equations gives $D \mathbf{A}=\mathbf{A A}$, which implies (take trace of both sides) that $\mathbf{A}=0$. Thus in an arbitrary dimension, as in $4 \mathrm{D}$, there are no non-expanding, twisting spacetimes.

(ii) If $A_{\max }=1$, i.e., $\lambda_{1}=L \neq 0$, then $\mathcal{M}_{A B C}=0, \mathcal{N}_{A B \gamma}=0$ and $\mathcal{A}_{A B}=0$. From (74) and (75), it follows $\mathcal{S}_{\alpha \beta \gamma}=0$ and $\mathcal{R}_{\alpha \beta C}=0$. Equations (80) and (76) give $\mathcal{P}_{A \beta \gamma}=0$. From (77) and (71), it follows $b_{\beta}=0$ and $\mathcal{O}_{A \beta A}=0$. Thus, the case $A_{\max }=1$ leads to a type $\mathrm{N}$ or $\mathrm{O}$ spacetime.

(iii) In the case $A_{\max }=2, \alpha_{\max }=1$ and thus, thanks to antisymmetry, $\mathcal{R}_{\alpha \beta C}=0$ and $\mathcal{S}_{\alpha \beta \gamma}=0$. Then from (80) $\mathcal{P}_{A \beta \gamma}=\mathcal{P}_{A \gamma \beta}$ and from (76) $\mathcal{P}_{B \alpha \gamma}=0$ or $\lambda_{B}=-L / 2$. Let us proceed further assuming that at least one non-vanishing $\mathcal{O}$ exists:

From (77) and (81), it follows that $\mathcal{O}_{112}=0$ is equivalent to $\mathcal{O}_{211}=0$. Similarly, from (77) and (71), $\mathcal{O}_{111}=0$ is equivalent to $\mathcal{O}_{212}=0$.

The case $\mathcal{O}_{112}, \mathcal{O}_{211} \neq 0$ was analyzed in Section 4 and leads to $\lambda_{1}=\lambda_{2}=L / 2$. If $\mathcal{O}_{112}=0=\mathcal{O}_{211}$ and $\mathcal{O}_{111}, \mathcal{O}_{212} \neq 0$ then (81) gives $\mathcal{N}_{A B \gamma}=0$ and from (77) and (71),

$$
b_{\beta}=\frac{\lambda_{1}-\lambda_{2}}{\lambda_{1}} \mathcal{O}_{1 \beta 1}=\frac{\lambda_{2}-\lambda_{1}}{\lambda_{2}} \mathcal{O}_{2 \beta 2}=-2\left(\mathcal{O}_{1 \beta 1}+\mathcal{O}_{2 \beta 2}\right),
$$

follows $\lambda_{1}=\lambda_{2}=L / 2$. Next we have to analyze possible cases with $\mathcal{O}_{A \beta C}=0$ $\forall A, \beta, C$.

Let us now assume that all $\mathcal{O}$ 's are zero and at least one $\mathcal{M}$ is non-vanishing. From (79) for $B=C$ and $B \neq A$ and from (72) we get

$$
\begin{aligned}
& \lambda_{2} a_{1}=2\left(3 \lambda_{1}-\lambda_{2}\right) \mathcal{M}_{122}, \quad\left(-3 \lambda_{1}+\lambda_{2}\right) a_{1}=-8 \lambda_{2} \mathcal{M}_{122}, \\
& \lambda_{1} a_{2}=2\left(3 \lambda_{2}-\lambda_{1}\right) \mathcal{M}_{211}, \quad\left(-3 \lambda_{2}+\lambda_{1}\right) a_{2}=-8 \lambda_{1} \mathcal{M}_{211} .
\end{aligned}
$$

If $a_{1} \neq 0$ (and thus also $\mathcal{M}_{122} \neq 0$ ) then the ratio of equations (C.22) gives $\left(3 \lambda_{1}+\lambda_{2}\right)\left(\lambda_{1}-\lambda_{2}\right)=0$ which has solutions

$$
\lambda_{2}=\lambda_{1} \text { or } \lambda_{2}=-3 \lambda_{1} \text {. }
$$


Similarly, if $a_{2} \neq 0, \mathcal{M}_{211} \neq 0$ then the ratio of equations (C.23) gives $\left(3 \lambda_{2}+\lambda_{1}\right)\left(\lambda_{2}-\lambda_{1}\right)=0$ with solutions $\lambda_{2}=\lambda_{1}$ or $\lambda_{1}=-3 \lambda_{2}$. Thus for $a_{1} \neq 0$ and $a_{2} \neq 0$, the only solution is $\lambda_{2}=\lambda_{1}=L / 2$.

If only one $a_{A} \neq 0$, without loss of generality $a_{1} \neq 0$ and $a_{2}=0$, then $\mathcal{M}_{211}=0$ and from (70) $\mathcal{P}_{211}=0$ and from (A.14) it follows $\mathcal{P}_{111}=0$. For the second solution in (C.24), $\lambda_{2}=-3 \lambda_{1}$, from (C.22) and (70) we obtain $a_{1}=-4 \mathcal{M}_{122}$ and $a_{1}=4 \mathcal{M}_{122}$, respectively, and thus this case does not occur.

If $a_{1}=0=a_{2}$ then (C.22), (C.23) imply that all $\mathcal{M}$ 's are zero and from (70) also all $\mathcal{P}$ 's vanish, which leads to a type $\mathrm{N}$ or $\mathrm{O}$ spacetime.

(iv) For $A_{\max }=3$, the only components of $\Psi_{i j k}, \Psi_{i}$ and $A_{i j}$ are $\mathcal{M}_{A B C}, a_{A}$ and $\mathcal{A}_{A B}$, respectively.

From (79) with $C=B$ we obtain the equation

$$
a_{A}=\left.\frac{2 L+4 \lambda_{A}-4 \lambda_{B}}{\lambda_{B}} \mathcal{M}_{A B B}\right|_{B \neq A}
$$

that together with (70) gives

$$
\begin{aligned}
& a_{1}=\frac{2 L+4 \lambda_{1}-4 \lambda_{2}}{\lambda_{2}} \mathcal{M}_{122}=\frac{2 L+4 \lambda_{1}-4 \lambda_{3}}{\lambda_{3}} \mathcal{M}_{133}=4\left(\mathcal{M}_{122}+\mathcal{M}_{133}\right), \\
& a_{2}=\frac{2 L+4 \lambda_{2}-4 \lambda_{1}}{\lambda_{1}} \mathcal{M}_{211}=\frac{2 L+4 \lambda_{2}-4 \lambda_{3}}{\lambda_{3}} \mathcal{M}_{233}=4\left(\mathcal{M}_{211}+\mathcal{M}_{233}\right), \\
& a_{3}=\frac{2 L+4 \lambda_{3}-4 \lambda_{1}}{\lambda_{1}} \mathcal{M}_{311}=\frac{2 L+4 \lambda_{3}-4 \lambda_{2}}{\lambda_{2}} \mathcal{M}_{322}=4\left(\mathcal{M}_{311}+\mathcal{M}_{322}\right)
\end{aligned}
$$

(A) If all $a_{A} \neq 0$ then the only solution is $\lambda_{1}=\lambda_{2}=L / 2$ and $\lambda_{3}=0$, which corresponds to $A_{\max }=2$ discussed above.

(B) Let us now study the case with one $a_{A}$ vanishing (we can assume $a_{3}=0$ without loss of generality). Then from (C.28) either $\mathcal{M}_{311}+\mathcal{M}_{322}=0$ and $\lambda_{1}=\lambda_{2}=L / 2+\lambda_{3}$, which implies $A_{\max }=2, \lambda_{3}=0, \lambda_{1}=\lambda_{2}=L / 2$, or $\mathcal{M}_{311}=\mathcal{M}_{322}=0$ and then from (C.26) and (C.27) we get

$$
\begin{aligned}
3 \lambda_{1}^{2}-\lambda_{2}^{2}-\lambda_{3}^{2}+2\left(-\lambda_{1} \lambda_{2}-\lambda_{1} \lambda_{3}+\lambda_{2} \lambda_{3}\right) & =0, \\
-\lambda_{1}^{2}+3 \lambda_{2}^{2}-\lambda_{3}^{2}+2\left(-\lambda_{1} \lambda_{2}+\lambda_{1} \lambda_{3}-\lambda_{2} \lambda_{3}\right) & =0 .
\end{aligned}
$$

Their difference gives

$$
4\left(\lambda_{1}-\lambda_{2}\right)\left(\lambda_{1}+\lambda_{2}-\lambda_{3}\right)=0
$$

and thus either $\lambda_{1}=\lambda_{2}$ and from (C.29) $\lambda_{3}=0$ or $\lambda_{3}=\lambda_{1}+\lambda_{2}$ and from (C.29) $\lambda_{1} \lambda_{2}=0$. This case thus again leads to $A_{\max }=2$.

(C) If only one $a_{A}$ is non-vanishing (we again choose $a_{1} \neq 0$ and $a_{2}=a_{3}=0$ without loss of generality) then (C.27), (C.28) imply either $\lambda_{1}=L / 2+\lambda_{3}=\lambda_{2}$ and $\lambda_{1}=L / 2+\lambda_{2}=\lambda_{3}$, which is not possible, or $\lambda_{1}=L / 2+\lambda_{3}=\lambda_{2}$, which gives $\lambda_{1}=\lambda_{2}=L / 2$ and $\lambda_{3}=0$, or $\lambda_{1}=L / 2+\lambda_{2}=\lambda_{3}$, which gives $\lambda_{1}=\lambda_{3}=L / 2$ and $\lambda_{2}=0$, or $\mathcal{M}_{211}=\mathcal{M}_{233}=\mathcal{M}_{311}=\mathcal{M}_{322}=0$. All these cases, except the last one are inconsistent with our assumption $A_{\max }=3$. We thus need to check the last case $\mathcal{M}_{211}=\mathcal{M}_{233}=\mathcal{M}_{311}=\mathcal{M}_{322}=0$, which has two possible branches corresponding to $\mathcal{M}_{123}=0$ and $\mathcal{M}_{123} \neq 0$.

(a) $\mathcal{M}_{123}=0$ : from (79) (in the form (C.14) $)$

$$
\begin{aligned}
\left(-\lambda_{1}+3 \lambda_{2}+\lambda_{3}\right) \mathcal{M}_{123} & =\left(-\lambda_{1}+\lambda_{2}+3 \lambda_{3}\right) \mathcal{M}_{132}, \\
\left(3 \lambda_{1}-\lambda_{2}+\lambda_{3}\right) \mathcal{M}_{213} & =\left(\lambda_{1}-\lambda_{2}+3 \lambda_{3}\right) \mathcal{M}_{231}, \\
\left(3 \lambda_{1}+\lambda_{2}-\lambda_{3}\right) \mathcal{M}_{312} & =\left(\lambda_{1}+3 \lambda_{2}-3 \lambda_{3}\right) \mathcal{M}_{321}
\end{aligned}
$$


we get $\lambda_{3}=0$ and $\lambda_{1}=L / 2=\lambda_{2}$ for $\mathcal{M}_{231}=\mathcal{M}_{132} \neq 0$, or $\mathcal{M}_{231}=\mathcal{M}_{132}=0$. Then from (A.6) we get $\mathcal{A}_{A B}=0$ and $a_{1}=\frac{4 \lambda_{1}}{\lambda_{2}} \mathcal{M}_{122}=\frac{4 \lambda_{1}}{\lambda_{3}} \mathcal{M}_{133}$ which together with (C.26) gives $\lambda_{2}=\lambda_{3}=L / 2, \lambda_{1}=0$.

(b) $\mathcal{M}_{123} \neq 0$ : from (C.32)

$$
\lambda_{1}^{2}+\lambda_{2}^{2}+\lambda_{3}^{2}-2\left(\lambda_{1} \lambda_{2}+\lambda_{1} \lambda_{3}+\lambda_{2} \lambda_{3}\right)=0
$$

with the solution $\lambda_{3}=\lambda_{1}+\lambda_{2} \pm 2 \sqrt{\lambda_{1} \lambda_{2}}$. By substituting this result into (C.29) we get $\lambda_{1}\left(\lambda_{2} \pm \sqrt{\lambda_{1} \lambda_{2}}\right)=0$ and thus either $\lambda_{1}=0$ and $\lambda_{2}=\lambda_{3}=L / 2$ or $\lambda_{1}=\lambda_{2}=L / 2$ and $\lambda_{3}=0\left(\lambda_{3}=4 \lambda_{1}\right.$ does not satisfy (C.29) $)$.

(D) If all $a_{A}=0$ then from (C.26) (C.28) either one of $\lambda_{A}=0$ and the other two are equal to $L / 2$ or all $\mathcal{M}_{A B B}=0$. Then if

a) $\mathcal{M}_{123}=0$ from (C.32) - (C.34) and (82) we get either one of $\lambda_{A}=0$ and other two are again equal to $L / 2$ or all $\mathcal{M}_{A B C}=0$, which corresponds to a type $\mathrm{N}$ or O spacetime.

b) If $\mathcal{M}_{123} \neq 0$ then from (C.32) - C.34) and (82) we get (C.35). Further from (A.6) we get all $\mathcal{A}_{A B}=0$ and then

$$
\begin{aligned}
& \lambda_{2} \mathcal{M}_{123}=\lambda_{3} \mathcal{M}_{132}, \\
& \lambda_{1} \mathcal{M}_{123}=-\lambda_{3} \mathcal{M}_{231}, \\
& \lambda_{1} \mathcal{M}_{132}=\lambda_{2} \mathcal{M}_{231},
\end{aligned}
$$

which yields

$$
\begin{aligned}
& 0=\left(\lambda_{2}-\lambda_{3}\right)\left(\lambda_{1}-\lambda_{2}-\lambda_{3}\right), \\
& 0=-\lambda_{1}^{2}+\lambda_{1} \lambda_{2}+3 \lambda_{1} \lambda_{3}+2 \lambda_{2} \lambda_{3}-2 \lambda_{3}^{2}, \\
& 0=-\lambda_{1}^{2}+3 \lambda_{1} \lambda_{2}+\lambda_{1} \lambda_{3}+2 \lambda_{2} \lambda_{3}-2 \lambda_{2}^{2},
\end{aligned}
$$

with the solution either $\lambda_{1}=\lambda_{2}+\lambda_{3}$ (then (C.40) implies $\lambda_{2}$ or $\lambda_{3}$ is zero) or $\lambda_{2}=\lambda_{3}$ and (C.40) implies $\lambda_{1}=0$ or $\lambda_{1}=4 \lambda_{2}$; however, from (A.18) we get $\left(\lambda_{2}-\lambda_{1}\right) \mathcal{M}_{123}=0$ which is contradiction.

\section{Appendix D. An example of a type $D$ vacuum spacetime}

The five-dimensional rotating black-hole metric in Boyer-Lindquist coordinates has the form [9, 10] (we use the notation of [10])

$$
\begin{aligned}
\mathrm{d} s^{2}= & \frac{\rho^{2}}{4 \Delta} \mathrm{d} x^{2}+\rho^{2} \mathrm{~d} \theta^{2}-\mathrm{d} t^{2}+\left(x+a^{2}\right) \sin ^{2} \theta \mathrm{d} \phi^{2}+\left(x+b^{2}\right) \cos ^{2} \theta \mathrm{d} \psi^{2} \\
& +\frac{r_{0}^{2}}{\rho^{2}}\left(\mathrm{~d} t+a \sin ^{2} \theta \mathrm{d} \phi+b \cos ^{2} \theta \mathrm{d} \psi\right)^{2},
\end{aligned}
$$

where

$$
\rho^{2}=x+a^{2} \cos ^{2} \theta+b^{2} \sin ^{2} \theta, \quad \Delta=\left(x+a^{2}\right)\left(x+b^{2}\right)-r_{0}^{2} x .
$$

After normalizing two null vectors $L_{+}$and $L_{-}$given in (4.23) in 10, one can appropriately choose the rest of the frame vectors $\boldsymbol{m}^{1}, \boldsymbol{m}^{2}$ and $\boldsymbol{m}^{3}$. It turns out that all components of the Weyl tensor with the boost weights 2, 1, -1, -2 vanish and hence the spacetime is of the algebraic type D 1,3 . One can also explicitly calculate the matrix $\boldsymbol{S}$. While the form of $\boldsymbol{S}$ depends on the choice of $\boldsymbol{m}^{1}, \boldsymbol{m}^{2}$ and $\boldsymbol{m}^{3}$, the characteristic polynomial of $\boldsymbol{S}, P_{\lambda}(\boldsymbol{S})$, does not. For $P_{\lambda}(\boldsymbol{S})$ we obtain

$$
P_{\lambda}(\boldsymbol{S})=\left(\lambda-\frac{1}{\sqrt{x}}\right)\left(\lambda-\frac{\sqrt{x}}{\rho^{2}}\right)^{2}
$$


and the diagonal form of $\boldsymbol{S}$ is

$$
\boldsymbol{S}_{\text {diag }}=\left(\begin{array}{ccc}
\frac{1}{\sqrt{x}} & 0 & 0 \\
0 & \frac{\sqrt{x}}{\rho^{2}} & 0 \\
0 & 0 & \frac{\sqrt{x}}{\rho^{2}}
\end{array}\right)
$$

From (20) it follows that shear is

$$
\sigma=\sqrt{\frac{2}{3 x}}\left(1-\frac{x}{\rho^{2}}\right)
$$

which is in accordance with equation (4.25) in [10]. Note that for $a=b=0$ all eigenvalues of $\boldsymbol{S}$ are equal and shear is thus zero. Whereas for $a^{2}+b^{2}>0$ the matrix $\boldsymbol{S}$ has two equal and one distinct eigenvalue and shear does not vanish. Note also that similarly as for the types III and N, this algebraically special vacuum solution has non-vanishing shear (see also footnote 4 in paper 10]). Moreover, in this spacetime $\boldsymbol{S}$ does not have properties proved in Sections 3 and 4 for vacuum type $\mathrm{N}$ and type III spacetimes.

\section{References}

[1] R. Milson, A. Coley, V. Pravda and A. Pravdová, Alignment and algebraically special tensors in Lorentzian geometry, gr-qc/0401010

[2] E. T. Newman and R. Penrose, An approach to gravitational radiation by a method of spin coefficients, J. Math. Phys. 3, 566 (1962).

[3] A. Coley, R. Milson, V. Pravda and A. Pravdová, Classification of the Weyl tensor in higher dimensions, Class. Quantum Grav. 21, L35 (2004).

[4] J. N. Goldberg and R. K. Sachs, A theorem on Petrov types, Acta. Phys. Polon., Suppl, 22, 13 (1962).

[5] H. Stephani, D. Kramer, M. MacCallum, C. Hoenselaers and E. Herlt, Exact Solutions to Einstein's Field Equations, Second Edition, Cambridge University Press, Cambridge (2003).

[6] V. Pravda, A. Pravdová, A. Coley and R. Milson, All spacetimes with vanishing curvature invariants, Class. Quantum Grav. 19, 6213 (2002).

[7] A. Coley, R. Milson, N. Pelavas, V. Pravda, A. Pravdová and R. Zalaletdinov, Generalizations of pp-wave spacetimes in higher dimensions, Phys. Rev. D 67, 104020 (2003).

[8] A. Coley, R. Milson, V. Pravda and A. Pravdová, Higher dimensional VSI spacetimes (in preparation).

[9] R. C. Myers and M. J. Perry, Black holes in higher dimensional space-times, Ann. Phys. 172, 304 (1986).

[10] V. Frolov and D. Stojković, Particle and light motion in space-time of a five-dimensional rotating black hole, Phys. Rev. D 68, 064011 (2003); V. Frolov and I. Novikov, Black hole Physics: Basic concepts and new developments, Kluwer, Dodrecht (1998). 